\title{
VARIABLE SELECTION CONSISTENCY OF GAUSSIAN PROCESS REGRESSION
}

\author{
By SHENG JiANG AND SURYA T. TOKDAR \\ Department of Statistical Science, Duke University, sheng.jiang@duke.edu; surya.tokdar@duke.edu \\ Bayesian nonparametric regression under a rescaled Gaussian process \\ prior offers smoothness-adaptive function estimation with near minimax- \\ optimal error rates. Hierarchical extensions of this approach, equipped with \\ stochastic variable selection, are known to also adapt to the unknown intrinsic \\ dimension of a sparse true regression function. But it remains unclear if such \\ extensions offer variable selection consistency, i.e., if the true subset of im- \\ portant variables could be consistently learned from the data. It is shown here \\ that variable consistency may indeed be achieved with such models at least \\ when the true regression function has finite smoothness to induce a polynomi- \\ ally larger penalty on inclusion of false positive predictors. Our result covers \\ the high dimensional asymptotic setting where the predictor dimension is al- \\ lowed to grow with the sample size. The proof utilizes Schwartz theory to \\ establish that the posterior probability of wrong selection vanishes asymptot- \\ ically. A necessary and challenging technical development involves providing \\ sharp upper and lower bounds to small ball probabilities at all rescaling levels \\ of the Gaussian process prior, a result that could be of independent interest.
}

1. Introduction. Sparse estimation and variable selection in high dimensional linear regression has been well studied [32, 6, 31, 21, 10, 17, 35, 33]. But an assumption of linearity could be overly restrictive and prone to model misspecification. A natural alternative is to allow the predictor-response relationship to be flexibly determined by an unknown smooth function $f: \mathbb{R}^{d} \rightarrow \mathbb{R}$, leading to the nonparametric regression model

$$
Y_{i}=f\left(X_{i}\right)+\epsilon_{i}, \quad \epsilon_{i} \mid X_{i} \stackrel{i i d}{\sim} N\left(0, \sigma^{2}\right),
$$

for paired data $\left(X_{i}, Y_{i}\right) \in \mathbb{R}^{d} \times \mathbb{R}, i=1, \ldots, n$. No theoretical results currently exist on simultaneously estimating $f$ and recovering its sparsity pattern, especially in the high dimensional setting. Earlier results restrict to the low dimensional settings of $d=o(\log n)$ or $d=O(\log n)$ $[36,19,3]$. Comminges and Dalalyan [11] present results under more relaxed settings, establishing that variable selection consistency is achievable for designs of size $\log (d)=o(n)$. However, they focus exclusively on understanding when it is possible to consistently recover the sparsity pattern of $f$, rather than providing a practicable estimation method of either the function or the sparsity pattern. More recent works have mostly concentrated on sparse additive formulations of $f[22,8]$.

In order to estimate $f$ from data, it is attractive to adopt a Bayesian approach where a Gaussian process prior is specified on the unknown regression function. Fairly expressive yet computationally tractable estimation models could be developed by specifying hierarchical extensions of this prior with rescaling and stochastic variable selection in order to infer smoothness and sparsity properties of $f$, and consequently, improve function estimation and prediction $[23,15]$. Indeed, under such Bayesian approaches, the posterior distribution of the

MSC2020 subject classifications: Primary 62G08, 62G20; secondary 60G05.

Keywords and phrases: Gaussian process priors, high-dimensional regression, nonparametric variable selection, Bayesian inference, adaptive estimation. 
regression function is known to contract to the true function at a near minimax optimal rate, adapting to both unknown smoothness and sparsity [29, 27, 4, 34].

Does this adaptive regression function estimation accuracy translate to correct identification of relevant regressors? We show that the answer is partially yes. Specifically, we establish that when the true sparsity pattern has a fixed cardinality $d_{0}$ and the true regression function is Sobolev $\beta$-smooth, an appropriately specified rescaled Gaussian process model with stochastic variable selection simultaneously estimates the regression function with near minimax optimal estimation accuracy and recovers the correct sparsity pattern with probability converging to one, provided $\log (d) \leq O\left(n^{d_{0} /\left(2 \beta+d_{0}\right)}\right)$ and the predictors are independent Gaussian variables with a common variance.

Like [11], our result covers the case where the predictor dimension may grow much faster than the sample size, but ties the growth rate with the smoothness level of the true regression function. Larger design dimensions are allowed when the true function is more rough. Both this smoothness dependent bound on the predictor dimension and the independent Gaussian design assumption are necessary for our proof technique which relies on establishing a polynomial slowdown in the posterior contraction rate of a rescaled Gaussian process model when it includes more predictors than necessary.

Establishing this polynomial slowdown in contraction rate requires new and detailed calculations of concentration probabilities of a rescaled Gaussian process around a given function of limited Sobolev smoothness. To prove that a rescaled Gaussian process estimation model achieves near minimax-optimal contraction rate, van der Vaart and van Zanten [29] derived lower bounds on the concentration probabilities for a carefully chosen range of rescaling levels. To establish a polynomial slowdown in rate, we additionally need sharp upper bounds on the concentration probabilities at every rescaling level. These are derived by an accurate characterization of small $L_{2}$-ball probabilities of a rescaled Gaussian process at every rescaling level. We use the metric entropy method [18, 20,28] to turn small ball probability calculations into calculating the metric entropy of the unit ball of the Reproducing Kernel Hilbert Space (RKHS) associated with the Gaussian process. Critically, the Gaussian design assumption allows mapping the RKHS of a squared-exponential Gaussian process to an $\ell_{2}$ ellipsoid whose metric entropy can be bounded accurately.

2. GP Regression with Stochastic Variable Selection. Our asymptotic analysis concerns a sequence of experiments, indexed by sample size $n=1,2, \ldots$, and with associated sample spaces $\mathcal{D}_{n}=\left\{\left(X_{i}, Y_{i}\right) \in \mathbb{R}^{d_{n}} \times \mathbb{R}, 1 \leq i \leq n\right\}$, in which $X_{i}$ 's are taken to be independent realizations from a probability measure $Q_{n}$ on $\mathbb{R}^{d_{n}}$ and $Y_{i}$ 's are realized as in the nonparametric regression formulation (1) for some known $\sigma>0$ and some unknown $f \in C\left(\mathbb{R}^{d_{n}}\right)$, the space of continuous functions on $\mathbb{R}^{d_{n}}$. We study a Bayesian estimation of $f$ under a rescaled Gaussian process prior with stochastic variable selection. This prior is formalized by hierarchically extending the rescaled Gaussian process prior of [29]; see also [34].

2.1. Prior specification. We call a stochastic process $W=\left(W(x): x \in \mathbb{R}^{d}\right)$ a standard, squared exponential Gaussian process on $\mathbb{R}^{d}$, if for any finite collection $\left\{x_{1}, \ldots, x_{k}\right\} \subset \mathbb{R}^{d}$, the random vector $\left(W\left(x_{1}\right), \ldots, W\left(x_{k}\right)\right)$ has a mean-zero, $k$-variate Gaussian distribution with covariance matrix $\left(\left(K\left(x_{i}, x_{j}\right)\right)_{1 \leq i, j \leq k}\right.$ where $K\left(x, x^{\prime}\right)=\exp \left\{-\left\|x-x^{\prime}\right\|_{2}^{2}\right\}$. It is well known that such a $W$ could be seen as a random element of $C\left(\mathbb{R}^{d}\right)$ and is infinitely differentiable with probability one.

Now, given a $\gamma \in\{0,1\}^{d}$ and a probability measure $\pi$ on $(0, \infty)$, define a $\gamma$-sparse, $\pi$ rescaled, squared exponential Gaussian process measure, $\operatorname{SEGP}\left(\mathbb{R}^{d} ; \gamma, \pi\right)$ for short, as the probability law of the stochastic process $W=\left(W(x): x \in \mathbb{R}^{d}\right)$ given as $W(x)=W_{0}\left(A x_{\gamma}\right)$ 
where $W_{0}$ is a standard, squared exponential Gaussian process on $\mathbb{R}^{|\gamma|}, A \sim \pi$ independently of $W_{0}$, and $x_{\gamma}=\left(x_{j}: \gamma_{j}=1, j=1, \ldots, d\right)$ denotes the sub-vector selected according to $\gamma$. The rescaling measure $\pi(A)$ plays a key role in smoothness adaptation, facilitating a Bayesian version of fully automated, data-driven bandwidth selection [29].

For our experiment sequence $\mathcal{D}_{n}$, a rescaled Gaussian process prior on $f \in C\left(\mathbb{R}^{d_{n}}\right)$ with stochastic variable selection is defined as the marginal law of $f$ induced by any joint probability measure $\Pi_{n}$ on $(\Gamma, f) \in\{0,1\}^{d_{n}} \times C\left(\mathbb{R}^{d_{n}}\right)$ satisfying the following

1. $\operatorname{Pr}(\Gamma=\gamma)=q_{n}(|\gamma|) /\left(\begin{array}{l}d_{n} \\ |\gamma|\end{array}\right), \gamma \in\{0,1\}^{d_{n}}$, for some probability vector $\left(q_{n}(d): 0 \leq d \leq d_{n}\right)$ with $q_{n}\left(d_{n}\right)<1$.

2. For every $\gamma \in\{0,1\}^{d_{n}}, f \mid(\Gamma=\gamma) \sim \operatorname{SEGP}\left(\mathbb{R}^{d_{n}} ; \gamma, \pi_{n,|\gamma|}\right)$, determined by a collection of probability measures $\pi_{n, d}$ on $(0, \infty), 0 \leq d \leq d_{n}$.

The sparsity pattern of $f$ is fully encoded by the binary vector $\Gamma$. Let $Q_{n, j}$ denote the marginal distribution of the $j$-th regressor under $X \sim Q_{n}$. Any $f \in C\left(\mathbb{R}^{d_{n}}\right)$ is constant along an axis $j \in\left\{1, \ldots, d_{n}\right\}$ if and only if $\left\|f-f_{j}\right\|_{L_{2}\left(Q_{n}\right)}=0$ where $f_{j}(x):=$ $\int f\left(x_{1}, \ldots, x_{j-1}, z, x_{j+1}, \ldots, x_{d_{n}}\right) d Q_{n, j}(z)$. Under the prior $\Pi_{n}$, the sparsity pattern of $f$ given by the subset $\left\{1 \leq j \leq d_{n}: f\right.$ is not constant along axis $\left.j\right\}$ is identical to $\Gamma$ with probability one. Notice that the prior on variable selection is taken to depend only on the cardinality of the included subset.

2.2. Connecting selection consistency with estimation accuracy. Given observed data $D_{n} \in \mathcal{D}_{n}$, let $\Pi_{n}\left(\cdot \mid D_{n}\right)$ denote the joint posterior distribution on $(\Gamma, f)$ under a prior distribution $\Pi_{n}$ as above. Assuming $D_{n}$ was generated from a true regression function $f_{n}^{*}$ whose sparsity pattern is identified by a $\gamma_{n}^{*} \in\{0,1\}^{d_{n}}$, the issue of variable selection consistency boils down to assessing whether $\Pi_{n}\left(\Gamma \neq \gamma_{n}^{*} \mid D_{n}\right) \rightarrow 0$ in some probabilistic manner. As indicated in the Introduction, the main result we prove in this paper is that when the cardinality of $\gamma_{n}^{*}$ remains fixed at a $d_{0}$ and $f_{n}^{*}$ is of finite Sobolev smoothness of order $\beta$, one has $\Pi_{n}\left(\Gamma \neq \gamma_{n}^{*} \mid D_{n}\right) \rightarrow 0$ as long as $\log \left(d_{n}\right) \precsim n^{d_{0} /\left(2 \beta+d_{0}\right)}$ and the regressors are independent Gaussian variables with equal variance. Below we give a sketch of the argument of how such a claim can be made based on adaptive function estimation accuracy.

The question of variable selection could be directly related to that of function estimation quality as follows. Consider a small ball around the truth: $E_{n}=\left\{f: \rho\left(f, f_{n}^{*}\right) \leq \varepsilon_{n}\right\}$ where $\rho$ is an appropriate metric and $\varepsilon_{n}>0$. Notice that

$$
\Pi_{n}\left(\Gamma \neq \gamma_{n}^{*} \mid D_{n}\right) \leq \Pi_{n}\left(f \in E_{n}^{c} \mid D_{n}\right)+\Pi_{n}\left(\Gamma \neq \gamma_{n}^{*}, f \in E_{n} \mid D_{n}\right) .
$$

If it were known that the posterior on $f$ contracts to the truth under metric $\rho$ at a rate $\varepsilon_{n}$ or faster, then the first term on the right hand side would eventually vanish in probability. In order for the second term to vanish as well, one needs to establish that the same fast rate of contraction cannot be achieved under a wrong selection of variables.

Partition the space of wrong selections into two parts: $\{0,1\}^{d_{n}} \backslash\left\{\gamma_{n}^{*}\right\}=\left\{\gamma \in\{0,1\}^{d_{n}}\right.$ : $\left.\gamma_{n}^{*} \not \leq \gamma\right\} \cup\left\{\gamma \in\{0,1\}^{d_{n}}: \gamma_{n}^{*}<\gamma\right\}=: \operatorname{FN}\left(\gamma_{n}^{*}\right) \cup \operatorname{FP}\left(\gamma_{n}^{*}\right)$, where the defining inequalities are taken to be coordinate-wise. The false negative set $\mathrm{FN}\left(\gamma^{*}\right)$ consists of selections that miss at least one true predictor. The false positive set $\mathrm{FP}\left(\gamma^{*}\right)$ contains selections that include all important variables and at least one unimportant regressor. Accordingly, the second posterior probability in (2) splits into two pieces,

$$
\begin{aligned}
\Pi_{n}\left(\Gamma \neq \gamma_{n}^{*}, f \in E_{n} \mid D_{n}\right)=\Pi_{n}(\Gamma & \left.\in \mathrm{FN}\left(\gamma_{n}^{*}\right), f \in E_{n} \mid D_{n}\right) \\
& +\Pi_{n}\left(\Gamma \in \mathrm{FP}\left(\gamma_{n}^{*}\right), f \in E_{n} \mid D_{n}\right),
\end{aligned}
$$

of which the first term could be expected to be exactly zero for large $n$ as long as one assumes that the signal strength of $f_{n}^{*}$ in any of its relevant variables, measured according to $\rho$, is above 
a fixed threshold $\delta_{n} \equiv \delta$ (Assumption 3 in Section 3). Such an $f_{n}^{*}$ will be at least a $\delta$ distance away in metric $\rho$ from any $f$ whose sparsity pattern $\gamma \in \operatorname{FN}\left(\gamma_{n}^{*}\right)$.

No such separation exists for regression functions associated with selections in the false positive set. For any $\gamma \in \operatorname{FP}\left(\gamma_{n}^{*}\right)$, one would expect the conditional posterior $\Pi_{n}(f \mid \Gamma=$ $\left.\gamma, D_{n}\right)$ to place considerable mass around the truth $f_{n}^{*}$. Any hope of the second term on the right hand side of (3) being small rests on establishing that such a conditional posterior would contract at a slower rate than $\varepsilon_{n}$. This is a legitimate hope because it is known that the minimax error rate of function estimation usually worsens when additional irrelevant variables are selected in the regression model [25]. Therefore, assuming $f_{n}^{*}$ belongs to the class of $\beta$ smooth functions for some $\beta>0$ (Assumption 4 part 1), a reasonable proof strategy would be to consider $\varepsilon_{n}=M \underline{\varepsilon}_{n}(\log n)^{\kappa}$ for some $M, \kappa>0$ where $\underline{\varepsilon}_{n}=n^{-\beta /\left(2 \beta+d_{0}\right)}$ is the usual minimax rate of estimation of an $f^{*}$ of $\beta$-smooth functions, and establish a polynomial difference in contraction rates between the overall posterior and the conditional posteriors under false positive selection.

In Sections 3 and 4 we establish the above rate difference result and give a formal proof of variable selection consistency under a rescaled GP prior with stochastic variable selection. Establishing slower posterior contraction rates under false positive selection necessitates working with the $L_{2}\left(Q_{n}\right)$ metric $\rho\left(f, f^{\prime}\right)=\left\|f-f^{\prime}\right\|_{L_{2}\left(Q_{n}\right)}=\left\{\int\left(f-f^{\prime}\right)^{2} d Q_{n}\right\}^{1 / 2}$. This choice of metric is slightly different than those considered in [29, 34]. Consequently, a new proof is needed to establish overall posterior contraction at a rate of $\varepsilon_{n}$. To show that posterior contraction rate becomes polynomially slower under false positive selection, we have to make two important assumptions: $f_{n}^{*}$ is exactly $\beta$-smooth and no smoother (Assumption 4 part 2) and $Q_{n}$ is the mean-zero Gaussian measure on $\mathbb{R}^{d_{n}}$ with covariance matrix $\xi^{2} \cdot I_{d_{n}}$ (Assumption 1). The finite smoothness of $f_{n}^{*}$ is indeed necessary to ensure that the conditional prior $\Pi(f \mid \Gamma=\gamma)$ sits less densely around $f_{n}^{*}$ when $\gamma \in \operatorname{FP}\left(\gamma_{n}^{*}\right)$ than when $\gamma=\gamma_{n}^{*}$. But the assumption of an independent Gaussian design with a fixed variance is a technical convenience that enables sharp calculations of the concentration probabilities of rescaled GP laws, which are necessary for establishing the above result. Additionally, the stochastic variable selection prior is assumed to favor small models, to control the total posterior probability of the exponentially growing false positive set (Assumption 7).

3. Main Result. Toward a formal and rigorous treatment of the arguments presented above, we first state the necessary assumptions and the main variable consistency result. A lengthy discussion of the assumptions is delayed to Section 6. Supporting results on the posterior contraction rates and difference in such rates under correct and false positive selections are presented in Section 4, relying upon the sharp small ball probability calculations in Section 5 .

An important assumption, needed essentially for technical reasons, is that the design distribution is uncorrelated Gaussian.

AsSUMPTION 1 (Gaussian random design). The design measure $Q_{n}=G_{d_{n}}$ where $G_{d}$ denotes the $d$-variate Gaussian measure $N_{d}\left(0, \xi^{2} I_{d}\right)$. The variance $\xi^{2}>2 / e$ may be unknown but does not change with $n$.

For each sample size $n$, the true data generating distribution $\mathbb{P}_{n}^{*}$ is taken to be an element of the model space identified by $f=f_{n}^{*} \in \mathcal{L}_{2}\left(Q_{n}\right)$ where $\mathcal{L}_{2}\left(Q_{n}\right):=C\left(\mathbb{R}^{d_{n}}\right) \cap L_{2}\left(Q_{n}\right)$.

Additionally, we assume that the sequence $\left(f_{n}^{*}: n \geq 1\right)$ remains essentially the same across $n$, formalized as below. For notational convenience, for any $d \in \mathbb{N}$ and $\gamma \in\{0,1\}^{d}$, let $T_{\gamma}: C\left(\mathbb{R}^{|\gamma|}\right) \rightarrow C\left(\mathbb{R}^{d}\right)$ denote the function embedding operator: $\left(T_{\gamma} f\right)(x)=f\left(x_{\gamma}\right)$, $f \in C\left(\mathbb{R}^{|\gamma|}\right), x \in \mathbb{R}^{d}$. With $T_{\gamma}$, any high dimensional functions with redundant variables 
can be decomposed into a low dimensional function without any redundant variables and a variable inclusion vector. Smoothness conditions are directly imposed to the low dimensional functions; sparsity and dimensionality assumptions are made on the variable inclusion vector.

ASSUMPTION 2 (Finite sparsity of true regression function). There exist $n_{0}, d_{0} \in \mathbb{N}$, $f_{0} \in \mathcal{L}_{2}\left(Q_{0}\right)$ and a sequence of binary vectors $\gamma_{n}^{*} \in\{0,1\}^{d_{n}}$, such that $d_{n} \geq d_{0},\left|\gamma_{n}^{*}\right|=d_{0}$ and $f_{n}^{*}=T_{\gamma_{n}^{*}} f_{0}$ for all $n \geq n_{0}$.

Assumption 2 makes it clear that for all large $n$, the true function is sparse and the support size $d_{0}$ does not grow with sample size. To avoid any ambiguity about the true sparsity level $d_{0}$, it is important to identify it as the minimal support size for the sequence $\left(f_{n}^{*}: n \geq 1\right)$. This is done via the next assumption on signal strength which ensures that each of the $d_{0}$ inputs to $f_{0}$ results in a variability that is detectable in the $L_{2}$ topology. Toward this, for each $j \in\left\{1, \ldots, d_{0}\right\}$, define $f_{0 j} \in \mathcal{L}_{2}\left(Q_{0}\right)$ as the projection of $f_{0}$ perpendicular to the $j$-th axis, given by $f_{0 j}(x):=\int_{\mathbb{R}} f_{0}\left(x_{1}, \ldots, x_{j-1}, z, x_{j+1}, \ldots, x_{d_{0}}\right) d G_{1}(z), x \in \mathbb{R}^{d_{0}}$.

Assumption 3 (Signal strength is $L_{2}$ detectable). The minimum signal strength in the relevant variables $\delta:=\min _{1 \leq j \leq d_{0}}\left\|f_{0}-f_{0 j}\right\|_{L_{2}\left(G_{d_{0}}\right)}^{2}$ is strictly positive.

An immediate consequence of Assumption 3 is that for any $n \geq n_{0}, f_{n}^{*}$ is at least a $\delta$ distance away from any $f \in \mathcal{L}_{2}\left(Q_{n}\right)$ that is constant along at least one axis $j$ for which $\gamma_{n, j}^{*}=1$. More formally, for any $n \geq n_{0}$, and any $\gamma \in\{0,1\}^{d_{n}}$ with $\gamma_{n}^{*} \not \leq \gamma$, inf $\left\{\| f_{n}^{*}-\right.$ $\left.f \|_{L_{2}\left(Q_{n}\right)}^{2}: f \in T_{\gamma} C\left(\mathbb{R}^{|\gamma|}\right) \cap \mathcal{L}_{2}\left(Q_{n}\right)\right\} \geq \delta$. To see why, without loss of generality, consider the toy example of $f_{0}\left(x_{1}, x_{2}\right)$ and $f\left(x_{1}, x_{3}\right)$ where $x_{1}$ and $x_{2}$ are correct variables and $x_{3}$ is the redundant variable. Then we can compute $\left\|f_{0}-f\right\|_{L_{2}(Q)}^{2}=\mathbb{E}\left[\left(f_{0}\left(X_{1}, X_{2}\right)-f\left(X_{1}, X_{3}\right)\right)^{2}\right]=$ $\mathbb{E}\left[\left(f_{0}\left(X_{1}, X_{2}\right)-f_{0,2}\left(X_{1}\right)+f_{0,2}\left(X_{1}\right)-f\left(X_{1}, X_{3}\right)\right)^{2}\right]$ whose cross-term $\mathbb{E}\left[\left(f_{0}\left(X_{1}, X_{2}\right)-\right.\right.$ $\left.\left.f_{0,2}\left(X_{1}\right)\right)\left(f_{0,2}\left(X_{1}\right)-f\left(X_{1}, X_{3}\right)\right)\right]=0$ holds because $X_{i}$ 's are independent.

Next, we formalize the notion that the true regression function is $\beta$-smooth but no smoother. For any $d \in \mathbb{N}, \beta>0$, let $H^{\beta}\left(\mathbb{R}^{d}\right)$ denote the Sobolev space of functions $h: \mathbb{R}^{d} \rightarrow$ $\mathbb{R}$ with norm $\|h\|_{H^{\beta}\left(\mathbb{R}^{d}\right)}$ given by

$$
\|h\|_{H^{\beta}\left(\mathbb{R}^{d}\right)}^{2}:=\int_{\mathbb{R}^{d}}|\hat{h}(\lambda)|^{2}\left(1+\|\lambda\|_{2}^{2}\right)^{\beta} d \lambda<\infty
$$

where $\hat{h}$ is the Fourier transform ${ }^{1}$ of $h$. Recall that functions $h \in H^{\beta}\left(\mathbb{R}^{d}\right)$ have squareintegrable, (weak) derivatives $D^{(k)} f, k=\left(k_{1}, \ldots, k_{d}\right) \in \mathbb{N}_{0}^{d}$, of order $|k| \leq \beta$.

ASSUMPTION 4 (Smoothness of $f_{0}$ ). The true function $f_{0}$ satisfies

1. There exists a $\beta>d_{0} / 2$ such that $f_{0} \in H^{\beta}\left(\mathbb{R}^{d_{0}}\right) \cap L_{2}\left(Q_{0}\right)$.

2. There also exists an $\alpha \in\left(\beta, \beta\left(1+1 / d_{0}\right)\right)$ such that $\left|\widehat{f_{0} \sqrt{g_{d_{0}}}}(\lambda)\right| \succsim\|\lambda\|_{2}^{-\left(\alpha+d_{0} / 2\right)}$ for every $\lambda \in \mathbb{R}^{d_{0}}$ with $\|\lambda\|_{2} \geq 1$, where $g_{d_{0}}$ is the probability density function of $G_{d_{0}}$.

Part 2 of the assumption ensures that $f_{0} \notin H^{b}\left(\mathbb{R}^{d_{0}}\right)$ for any $b>\alpha$ and hence has limited regularity which is important in establishing that the posterior contraction rate at $f_{n}^{*}$ is polynomially slower under false positive inclusion.

The posterior contraction rates also depend on the rescaling measures $\pi_{n, d}, 0 \leq d \leq d_{n}$. The following assumption is mildly adapted from [29]. The modification is needed in part

$$
{ }^{1} \hat{h}(\lambda)=(2 \pi)^{-d} \int_{\mathbb{R}^{d}} e^{-i(\lambda, t)} h(t) d t, \lambda \in \mathbb{R}^{d} .
$$


because in determining sharp upper bounds on the concentration probabilities of rescaled GP priors, one needs to integrate over the entire range of the rescaling parameter. Below, with a slight abuse of notation, we let $\pi_{n, d}$ also denote the probability density function underlying the eponymous rescaling measure.

Assumption 5 (Rescaling measures). For each $d \in \mathbb{N}$, there exist constants $C_{1}, C_{2}, C_{3}$, $D_{1}$ and $D_{2}$, all independent of $d$, such that

1. for all sufficiently large $a, \pi_{n, d}(a) \geq D_{1} e^{-C_{1} a^{d} \log ^{d+1}(a)}$;

2. for every $a>1 / \xi, \pi_{n, d}(a) \leq D_{2} a^{d-1} e^{-C_{2} a^{d}\left(\log ^{d+1}(a) \vee 1\right)+C_{3} \log (d)}$;

3. $\pi_{n, d}\left(0, \xi^{-1}\right)=0$.

This assumption is satisfied, for example, when $\pi_{n, d}$ is the truncation to $\left(\xi^{-1}, \infty\right)$ of the probability law of a random variable $A$ for which $A^{d} \log ^{d+1}(A)$ has an exponential distribution with a rate parameter that is constant in $n$ and $d$.

Our next assumption regulates how fast the design dimension $d_{n}$ can grow in $n$. If the support $\gamma_{n}^{*}$ were known, the $L_{2}\left(Q_{n}\right)$ minimax estimation error of a $\beta$-smooth function would be of the order of $\underline{\varepsilon}_{n}=n^{-1 /\left(2+d_{0} / \beta\right)}$ [25]. Not knowing the support means that one incurs an additional error of the order of $d_{0} \log \left(d_{n} / d_{0}\right) / n$ for having to carry out variable selection. We require this additional error to not overwhelm the original estimation error.

ASSUMPTION 6 (Growth of $d_{n}$ ). The design dimension $d_{n}$ satisfies $\log \left(d_{n}\right) \precsim n \underline{\varepsilon}_{n}^{2} \asymp$ $n^{d_{0} /\left(2 \beta+d_{0}\right)}$.

A final assumption is needed on the sparsity induced by the prior distribution. In particular it is needed that the prior on $\Gamma$ favors small selection sizes and heavily penalizes extremely large selections.

ASSUMPTION 7 (Prior sparsity). For all sufficiently large $n$,

1. $q_{n}\left(d_{0}\right) \geq \exp \left\{-n \underline{\varepsilon}_{n}^{2}\right\} \asymp \exp \left\{-n^{d_{0} /\left(2 \beta+d_{0}\right)}\right\}$

2. $q_{n}(d) \leq \exp \left\{-C \bar{d}^{\rho}\right\}$ for every $d \succsim n^{2 \beta /\left\{\alpha\left(2 \beta+d_{0}\right)\right\}}$, for some constants $C>0$ and $\rho \geq$ $\left(d_{0}+1\right) / 2$.

Assumption 7 seemingly requires the knowledge of the true support size $d_{0}$, but one can relax this by letting $\rho$ grow slowly as sample size increases. The assumption would hold, for instance if one chose a prior that caps the selection size $|\Gamma|$ at an $m_{n} \leq d_{n}$ and let $m_{n}$ grow slowly with $n$, e.g., $m_{n} \asymp n^{1 / \log \log n}$. Formally, $q_{n}(d) \propto I\left(d<n^{1 / \log \log n}\right), d=0, \ldots, d_{n}$. An alternative is to not use a cap, but employ aggressive penalization of larger selections: $q_{n}(d) \propto d^{k \log \log n-1} \exp \left\{-d^{k \log \log n}\right\}, 0 \leq d \leq d_{n}$, for some constant $k$. The latter choice is equivalent to an appropriately tuned Beta-Binomial prior on individual regressor inclusion.

Building upon these formal assumptions, we are able to offer the following rigorous statement and proof of variable selection consistency.

TheOREm 3.1. Under Assumptions 1-7, $\mathbb{P}_{n}^{*}\left[\Pi_{n}\left(\Gamma \neq \gamma_{n}^{*} \mid D_{n}\right)\right] \rightarrow 0$, as $n \rightarrow \infty$.

PROOF. As before, let $E_{n}=\left\{f \in \mathcal{L}_{2}\left(Q_{n}\right):\left\|f-f_{n}^{*}\right\|_{L_{2}\left(Q_{n}\right)} \leq \varepsilon_{n}\right\}$ where $\varepsilon_{n}=\underline{\varepsilon}_{n}(\log n)^{\kappa}$ with $\kappa=\left(d_{0}+1\right) /\left(2+d_{0} / \beta\right)$. Consider the upper bound on $\Pi_{n}\left(\Gamma \neq \gamma_{n}^{*} \mid D_{n}\right)$ jointly given in (2) and (3). By Theorem 4.1 in the next section, $\Pi_{n}\left(f \in E_{n}^{c} \mid D_{n}\right) \rightarrow 0$ in probability as $n \rightarrow \infty$. By Assumption 3, the first term of the bound given by (3) is exactly zero for all large $n$ because the prior probability $\Pi_{n}\left(\Gamma \in \mathrm{FN}\left(\gamma_{n}^{*}\right), f \in E_{n}\right)=0$ whenever $\varepsilon_{n}<\delta$. The second piece of this bound vanishes in probability by Proposition 1, which leverages on detailed calculations of concentration properties of Gaussian process laws presented in Section 5. 
4. Posterior Concentration via Schwartz Theory. This Section presents supporting results for the proof of Theorem 3.1. As the proof technique is standard, details of the proofs are in the Supplement.

THEOREM 4.1. Under Assumptions 1, 2, 4-1, 6 and 5, let $\varepsilon_{n}=\underline{\varepsilon}_{n}(\log n)^{\kappa}$ with $\kappa=$ $\left(d_{0}+1\right) /\left(2+d_{0} / \beta\right)$ for $n \geq 1$, then for any sufficiently large constant $M$,

$$
\left.\mathbb{P}_{n}^{*}\left[\Pi_{n}\left(f \in \mathcal{L}_{2}\left(Q_{n}\right):\left\|f-f_{n}^{*}\right\|_{L_{2}\left(Q_{n}\right)}>M \varepsilon_{n}\right\} \mid D_{n}\right)\right] \rightarrow 0 \text {, as } n \rightarrow \infty .
$$

A proof of this result is presented in the Supplement by verifying Theorem 2.1 of [12]. In the proof, we first verify the Kullback-Leibler prior mass condition that for all sufficiently large $n$,

$$
\Pi_{n}\left(f \in B_{n}\left(f_{n}^{*}, \varepsilon_{n}\right)\right) \geq e^{-n \varepsilon_{n}^{2}}
$$

where for any $g \in \mathcal{L}_{2}\left(Q_{n}\right)$ and $\epsilon>0$, one defines $B_{n}(g, \epsilon)=\left\{f: K\left(\mathbb{P}_{g}^{1}, \mathbb{P}_{f}^{1}\right) \leq \epsilon^{2}, V\left(\mathbb{P}_{g}^{1}, \mathbb{P}_{f}^{1}\right) \leq\right.$ $\left.\epsilon^{2}\right\}$, with $\mathbb{P}_{f}^{1}$ denoting the probability distribution of a single observation pair $\left(X_{1}, Y_{1}\right)$ under the model element with regression function $f$, and, $K\left(\mathbb{P}_{g}^{1}, \mathbb{P}_{f}^{1}\right)=\mathbb{P}_{g}^{1} \log \left(d \mathbb{P}_{g}^{1} / d \mathbb{P}_{f}^{1}\right)$, $V\left(\mathbb{P}_{g}^{1}, \mathbb{P}_{f}^{1}\right)=\mathbb{P}_{g}^{1}\left[\left(\log \left(d \mathbb{P}_{g}^{1} / d \mathbb{P}_{f}^{1}\right)\right)^{2}\right]-K\left(\mathbb{P}_{g}^{1}, \mathbb{P}_{f}^{1}\right)^{2}$. Next, a sieve $\mathbb{B}_{n} \subset L_{2}\left(Q_{n}\right), n \in \mathbb{N}$ is produced such that $\Pi_{n}\left(f \notin \mathbb{B}_{n}\right) \leq \exp \left(-4 n \varepsilon_{n}^{2}\right)$ and $\log N\left(\varepsilon_{n}, \mathbb{B}_{n},\|\cdot\|_{L_{2}\left(Q_{n}\right)}\right) \leq n \varepsilon_{n}^{2}$ for all sufficiently large $n$. Here, $N(\epsilon, S, \rho)$ is used to denote the $\epsilon$-covering number of a subset $S$ in a metric space with metric $\rho$.

Our statement and proof technique for Theorem 4.1 mirror the near minimax-optimal posterior contraction results and proofs of [29, 34], but are slightly novel in its use of the $L_{2}\left(Q_{n}\right)$ topology on the space of regression functions. In particular, under the stochastic design assumption, one has the simplification that $\left.B_{n}(g, \epsilon)=\left\{f \in \mathcal{L}_{2}\left(Q_{n}\right):\|f-g\|_{L_{2}\left(Q_{n}\right)} \leq \epsilon\right\}\right)$. Thus the Kullback-Leibler prior mass condition translates to a simpler $L_{2}\left(Q_{n}\right)$ prior mass condition:

$$
\Pi_{n}\left(\left\{f \in \mathcal{L}_{2}\left(Q_{n}\right):\left\|f-f_{n}^{*}\right\|_{L_{2}\left(Q_{n}\right)} \leq \varepsilon_{n}\right\}\right) \geq e^{-n \epsilon_{n}^{2}} .
$$

Proposition 1. Under Assumptions 1, 2, 4-2, 5 and 7, let $E_{n}=\left\{f \in \mathcal{L}_{2}\left(Q_{n}\right): \| f-\right.$ $\left.f_{n}^{*} \|_{L_{2}\left(Q_{n}\right)} \leq M \varepsilon_{n}\right\}, \varepsilon_{n}=\underline{\varepsilon}_{n}(\log n)^{\kappa}$ with $\kappa=\left(d_{0}+1\right) /\left(2+d_{0} / \beta\right)$ for $n \geq 1$, then for all sufficiently large constant $M$,

$$
\mathbb{P}_{n}^{*}\left[\Pi_{n}\left(\Gamma \in \operatorname{FP}\left(\gamma_{n}^{*}\right), f \in E_{n} \mid D_{n}\right)\right] \rightarrow 0, \text { as } n \rightarrow \infty .
$$

A proof is given in the Supplement. It follows Lemma 1 of [9] and establishes that

$$
\frac{\Pi_{n}\left(\Gamma \in \mathrm{FP}\left(\gamma_{n}^{*}\right), f \in E_{n}\right)}{\Pi_{n}\left(f \in B_{n}\left(f_{n}^{*}, \varepsilon_{n}\right)\right)} \leq e^{-2 n \varepsilon_{n}^{2}},
$$

which, according to Lemma 1 in [14], along with (5), guarantees that the posterior probability of the set in the numerator vanishes in probability.

The message of Proposition 1 is that the posterior contraction rates of false positive models are significantly slower than minimax rates. With Theorem 4.1, it further implies false positive models eventually receive negligible posterior mass. The use of posterior contraction rates adaptation echoes [13] who establishes model selection consistency among density models with different regularity levels.

The key inequalities in (6) and (7) require establishing lower and upper bounds on the prior concentration in $L_{2}\left(Q_{n}\right)$ balls around $f_{n}^{*}$. Such concentrations are completely governed by the $f_{n}^{*}$-shifted small ball probabilities of the underlying sparse Gaussian processes at appropriate rescaling levels. The lower bound calculations are similar to those in [29] and 
require working with rescaling levels that appropriately grow to infinity as determined by the true smoothness level $\beta$. But, as mentioned earlier, the upper bound calculations are much more technically involved and require dealing with all rescaling levels. Furthermore, unlike [29] we calculate small ball probabilities by viewing the Gaussian process as a random element in $L_{2}\left(Q_{n}\right)$, necessitating new characterization of the associated reproducing kernel Hilbert space. These details are presented in the next section.

\section{Small Ball Probability of Rescaled GP.}

5.1. Series representation of $W^{a, \gamma}$. Let $W^{a, \gamma} \sim \operatorname{SEGP}\left(\mathbb{R}^{d_{n}} ; \gamma, \delta_{a}\right)$ where $\gamma \in\{0,1\}^{d_{n}}$, $|\gamma|>0$, and $a>0$ is a fixed rescaling level, i.e., the rescaling measure is the Dirac delta measure at level $a$. In this section, we obtain a series representation of $W^{a, \gamma}$ via the KarhunenLoève expansion of the covariance kernel of $W^{a, \gamma}$.

The covariance kernel of $W^{a, \gamma}$ is

$$
K_{a, \gamma}(s, t)=e^{-a^{2}\left\|s_{[\gamma]}-t_{[\gamma]}\right\|_{2}^{2}}=\prod_{\left\{i: \gamma_{i}=1\right\}} e^{-a^{2}\left(s_{i}-t_{i}\right)^{2}} .
$$

To obtain an eigen-expansion of $K_{a, \gamma}(\cdot, \cdot)$, first recall the eigen-expansion of univariate Squared Exponential kernel function under Gaussian design. For $s, t \in \mathbb{R}, K_{a, 1}(s, t)=$ $e^{-a^{2}(s-t)^{2}}=\sum_{j=0}^{\infty} \lambda_{j} \varphi_{j}(s) \overline{\varphi_{j}(t)}$, where eigenvalues $\lambda_{j}=\sqrt{2 v_{1} / V} B^{j}$, eigenfunctions $\varphi_{j}(x)=e^{-\left(v_{3}-v_{1}\right) x^{2}} H_{j}\left(\sqrt{2 v_{3}} x\right)$ with physicists' Hermite polynomial $H_{j}(x)=(-1)^{j} e^{x^{2}} \frac{d^{j}}{d x^{j}} e^{-x^{2}}$, and the constants in the eigen-expansion are defined as follows,

$$
v_{1}^{-1}=4 \xi^{2}, v_{2}=a^{2}, v_{3}=\sqrt{v_{1}^{2}+2 v_{1} v_{2}}, V=v_{1}+v_{2}+v_{3}, B=v_{2} / V .
$$

(See Chapter 4.3 of [23] for more details). The eigenfunctions $\left\{\varphi_{j}\right\}$ form an orthonormal basis under the inner product

$$
\left\langle\varphi_{j}, \varphi_{k}\right\rangle_{G}=\int_{\mathbb{R}} \varphi_{j}(s) \varphi_{k}(s) g(s) d s=\delta_{0}(j-k),
$$

where $G$ denotes the univariate Normal distribution $N\left(0, \xi^{2}\right)$ and $g$ is its density function relative to Lebesgue measure. The Gaussian measure $G$ corresponds to Assumption 1.

With the univariate expansion, $K_{a, \gamma}$ is a tensor product of univariate SE kernels and admits the following expansion: for $s, t \in \mathbb{R}^{d_{n}}$,

$$
\begin{aligned}
K_{a, \gamma}(s, t) & =\prod_{\left\{i: \gamma_{i}=1\right\}}\left(\sum_{j=0}^{\infty} \lambda_{j}^{(i)} \varphi_{j}^{(i)}\left(s_{i}\right) \overline{\varphi_{j}^{(i)}\left(t_{i}\right)}\right) \\
& \equiv \sum_{k=0}^{\infty} \mu_{k}^{(\gamma)} \psi_{k}^{(\gamma)}(s) \overline{\psi_{k}^{(\gamma)}(t)},
\end{aligned}
$$

where $\lambda_{j}^{(i)}$ is the $j^{\text {th }}$ eigenvalue of $i^{\text {th }}$ univariate SE kernel, $\varphi_{j}^{(i)}$ is the $j^{\text {th }}$ eigenfunction of $i^{\text {th }}$ univariate SE kernel, eigenfunctions $\left\{\psi_{k}^{(\gamma)}\right\}$ and eigenvalues $\left\{\mu_{k}^{(\gamma)}\right\}$ are ordered by collecting lower order terms first.

The eigenvalue $\mu_{k}^{(\gamma)}=\left(2 v_{1} / V\right)^{|\gamma| / 2} B^{m}$ for some $m \in \mathbb{N}$ and $m$ is weakly increasing in $k$. Note the number of $k$-tuples of positive integers whose sum is $m$ is $\left(\begin{array}{c}m-1 \\ k-1\end{array}\right)$, the number of terms involving $B^{m}$ for $m \geq 1$ is $\sum_{k=1}^{m}\left(\begin{array}{c}m-1 \\ k-1\end{array}\right)\left(\begin{array}{c}|\gamma| \\ k\end{array}\right)=\left(\begin{array}{c}|\gamma|+m-1 \\ m\end{array}\right)$ by Vandermonde's identity. By ratio test, the eigenvalues are summable: $\sum_{m=1}^{\infty}\left(\begin{array}{c}|\gamma|+m-1 \\ m\end{array}\right) B^{m}<\infty$. As we collect low order terms first, first few terms are $\mu_{0}^{(\gamma)}=\left(2 v_{1} / V\right)^{|\gamma| / 2}, \mu_{k}^{(\gamma)}=\left(2 v_{1} / V\right)^{|\gamma| / 2} B$ for $k=1$ : $|\gamma|, \mu_{k}^{(\gamma)}=\left(2 v_{1} / V\right)^{|\gamma| / 2} B^{2}$ for $k=|\gamma|+1:|\gamma|+\left(\begin{array}{c}|\gamma|+1 \\ 2\end{array}\right)$. (Recall the constants in (9).) 
Eigenfunctions are defined accordingly depending on eigenvalues. The ordering of the eigenfunctions with the same eigenvalues does not matter. The eigenfunctions are orthogonal if the base measure is $Q_{n} \equiv N\left(0, \xi^{2} I_{d_{n}}\right)$ where isotropy is assumed without loss of generality.

With the eigen-expansion of $K_{a, \gamma}(\cdot, \cdot)$ and the summable eigenvalues, $W^{a, \gamma}$ has the series representation: for $Z_{j} \stackrel{i i d}{\sim} N(0,1), j=0,1, \ldots$,

$$
W_{t}^{a, \gamma}=\sum_{j=0}^{\infty} Z_{j} \sqrt{\mu_{j}^{(\gamma)}} \psi_{j}^{(\gamma)}(t)
$$

By orthonormality of $\left\{\psi_{j}^{(\gamma)}\right\},\left\|W^{a, \gamma}\right\|_{L_{2}\left(Q_{n}\right)}^{2}=\sum_{j=0}^{\infty} Z_{j}^{2} \mu_{j}^{(\gamma)}$. Then, $W^{a, \gamma} \in L_{2}\left(Q_{n}\right)$, a.s..

5.2. Concentration Function. With the series representation (11), we can treat the Gaussian process $W^{a, \gamma}$ as a Borel measurable map into the Banach space $\left(L_{2}\left(Q_{n}\right),\|\cdot\|_{L_{2}\left(Q_{n}\right)}\right)$ such that the random variable $b^{*}\left(W^{a, \gamma}\right)$ is Gaussian for every continuous, linear map $b^{*}: L_{2}\left(Q_{n}\right) \rightarrow \mathbb{R}$. Since $W^{a, \gamma}$ is continuous with probability 1 , the sample paths under consideration are continuous versions. For any set $U \subset L_{2}\left(Q_{n}\right)$, the probability $\operatorname{Pr}\left(W^{a, \gamma} \in U\right)$ equals the probability $\operatorname{Pr}\left(W^{a, \gamma} \in U \cap C\left(\mathbb{R}^{d_{n}}\right)\right)$. In the end, the Banach space where $W^{a, \gamma}$ lives is essentially $\mathcal{L}_{2}\left(Q_{n}\right) \equiv L_{2}\left(Q_{n}\right) \cap C\left(\mathbb{R}^{d_{n}}\right)$.

The RKHS $\mathcal{H}^{a, \gamma}$ associated with $W^{a, \gamma}$ is the completion of the range $S\left[\mathcal{L}_{2}\left(Q_{n}\right)^{*}\right]$ where $S\left[b^{*}\right]=\mathbb{E}\left[W^{a, \gamma} b^{*}\left(W^{a, \gamma}\right)\right], b^{*} \in \mathcal{L}_{2}\left(Q_{n}\right)^{*}$ in the sense of Pettis integral; see [28] for more details. The corresponding RKHS norm is determined by the inner product $\left\langle S b_{1}^{*}, S b_{2}^{*}\right\rangle_{\mathcal{H}^{a, \gamma}}=$ $\mathbb{E}\left(b_{1}^{*}\left(W^{a, \gamma}\right) b_{2}^{*}\left(W^{a, \gamma}\right)\right)$. Using the same argument as (2.4) of [28], the RKHS norm is stronger than the $L_{2}\left(Q_{n}\right)$ norm. Hence, $\mathcal{H}^{a, \gamma}$ is seen as a dense subset of $\mathcal{L}_{2}\left(Q_{n}\right)$ and can be isometrically identified with an $\ell_{2}$ sequence space. In our case, with the eigen-expansion (10), the RKHS unit ball $\mathcal{H}_{1}^{a, \gamma}$ of $W^{a, \gamma}$ can be isometrically identified with the following ellipsoid:

$$
\left\{\left\{\theta_{j}\right\}_{j=1}^{\infty}: \sum_{j=1}^{\infty} \theta_{j}^{2} / \mu_{j}^{(\gamma)} \leq 1\right\} \subseteq \ell^{2}(\mathbb{N})
$$

This isometry provides a different route to compute the metric entropy of the unit ball of $\mathcal{H}^{a, \gamma}$

Prior mass calculations in Theorem 4.1 and Proposition 1 require both upper and lower bounds for $\operatorname{Pr}\left(\left\|W^{a, \gamma}-f_{n}^{*}\right\|_{L_{2}\left(Q_{n}\right)}<\varepsilon_{n}\right)$, the $f_{n}^{*}$-shifted $\varepsilon_{n}$-ball probability of $W^{a, \gamma}$.

With the above abstract formulation, the small ball probability, in log scale, can be bounded as

$$
\phi_{f_{n}^{*}}^{a, \gamma}\left(\varepsilon_{n}\right) \leq-\log \operatorname{Pr}\left(\left\|W^{a, \gamma}-f_{n}^{*}\right\|_{L_{2}\left(Q_{n}\right)}<\varepsilon_{n}\right) \leq \phi_{f_{n}^{*}}^{a, \gamma}\left(\varepsilon_{n} / 2\right),
$$

with $\phi_{f_{n}^{*}}^{a, \gamma}$ denoting the concentration function

$$
\phi_{f_{n}^{*}}^{a, \gamma}\left(\varepsilon_{n}\right)=\inf _{h \in \mathcal{H}^{a, \gamma}:\left\|h-f_{n}^{*}\right\|_{L_{2}\left(Q_{n}\right)}<\varepsilon_{n}}\|h\|_{\mathcal{H}^{a, \gamma}}^{2}-\log \operatorname{Pr}\left(\left\|W^{a, \gamma}\right\|_{L_{2}\left(Q_{n}\right)}<\varepsilon_{n}\right),
$$

where $\|\cdot\|_{\mathcal{H}^{a, \gamma}}$ is the canonical norm of the Hilbert space $\mathcal{H}^{a, \gamma}$.

With inequality (13), bounding shifted small ball probability is essentially bounding the concentration function. The concentration function has two parts: the decentering part and the centered small ball probability exponent. The decentering part measures the position of the centering $f_{n}^{*}$ relative to the RKHS.

5.3. Centered Small Ball Probability Bounds via Metric Entropy. The centered small ball probability is bounded using the metric entropy method [18, 20, 28]. The metric entropy method links the metric entropy of the RKHS unit ball with the centered small ball probability. Section 6 of the review paper [28] summarizes the quantitative relationship between 
bounds of metric entropy and bounds of small ball probability. In our analysis, we first calculate the metric entropy of the RKHS unit ball and then use the relationship to derive bounds of centered small ball probabilities as a corollary.

With isometry, the metric entropy of the RKHS unit ball is the metric entropy of the $\ell_{2}$ ellipsoid (12). It is well known that the metric entropy of $\ell_{2}$ ellipsoid in the fashion of (12) depends on the decay rate of $\left\{\mu_{j}^{(\gamma)}\right\}$. Lemma 5.1 gives bounds for the metric entropy of the RKHS unit ball.

LEMMA 5.1. Suppose $\mathcal{H}_{1}^{a, \gamma}$ is the RKHS unit ball associated to the GP SEGP $\left(\mathbb{R}^{d_{n}} ; \gamma, \delta_{a}\right)$ with the design measure $Q_{n} \equiv N\left(0, \xi^{2} I_{d_{n}}\right)$, constants $v_{1}$ and $V$ are defined in (9), constants $a$, $\varepsilon$ and $|\gamma|$ satisfy $\varepsilon^{-2} \geq C_{H}(a \xi)^{|\gamma|}$ for some constant $C_{H}$ such that $\log \left(\frac{1}{\varepsilon}\right)-\frac{|\gamma|}{4} \log \left(\frac{V}{2 v_{1}}\right) \asymp$ $\log \left(\frac{1}{\varepsilon}\right)$, and a $\xi \log (1 / \varepsilon)>|\gamma|$, then the metric entropy of $\mathcal{H}_{1}^{a, \gamma}$ satisfies

$$
\begin{aligned}
& \log N\left(\mathcal{H}_{1}^{a, \gamma}, \varepsilon,\|\cdot\|_{L_{2}\left(Q_{n}\right)}\right) \precsim a^{|\gamma|} \log (1 / \varepsilon)^{|\gamma|+1} /|\gamma| !, \\
& \log N\left(\mathcal{H}_{1}^{a, \gamma}, \varepsilon,\|\cdot\|_{L_{2}\left(Q_{n}\right)}\right) \succsim a^{|\gamma|} \log (1 / \varepsilon)^{|\gamma|} /|\gamma| ! \text {. }
\end{aligned}
$$

The proof of Lemma 5.1 follows standard technique of metric entropy of $\ell_{2}$ sequence spaces. The lower bound is smaller than the upper bound by a logarithmic factor. This gap affects the bounds for small ball probabilities, but it does not jeopardize Theorem 4.1 and Proposition 1.

Then the centered small ball probability bounds are obtained in Lemma 5.2 as a corollary.

LEMMA 5.2. Suppose $\phi_{0}^{a, \gamma}$ is the concentration function associated to the GP SEGP $\left(\mathbb{R}^{d_{n}} ; \gamma, \delta_{a}\right)$ with the design measure $Q_{n} \equiv N\left(0, \xi^{2} I_{d_{n}}\right)$, constants a and $\varepsilon$ satisfy $\varepsilon^{-2} \geq C_{H}(a \xi)^{|\gamma|}$ and $a \xi \log (1 / \varepsilon)>|\gamma|$, then there exists a constant $C$ independent of $a, \xi$ and $|\gamma|$ such that

$$
\phi_{0}^{a, \gamma}(\varepsilon) \leq C a^{|\gamma|} \log (a / \varepsilon)^{|\gamma|+1} /|\gamma| !,
$$

and there exists a constant $C^{\prime}$ such that

$$
\phi_{0}^{a, \gamma}(\varepsilon) \geq C^{\prime} a^{|\gamma|} \log (1 / \varepsilon)^{|\gamma|} /|\gamma| !
$$

PROOF. With the assumptions, the metric entropy calculation in Lemma 5.1 holds. Following the same idea as in Lemma 4.6 of [29], the first assertion holds.

Using the first inequality of Lemma 2.1 in [1], let $\lambda=2$ in the Lemma, $\phi_{0}^{a, \gamma}\left(\varepsilon_{n}\right) \geq$ $\log N\left(\mathcal{H}^{a, \gamma}, \varepsilon_{n},\|\cdot\|_{L_{2}\left(Q_{n}\right)}\right)-2 \geq C^{\prime} a^{|\gamma|} \log (1 / \varepsilon)^{|\gamma|} /|\gamma| !$ holds for some constant $C^{\prime}$.

5.4. Shifted Small Ball Probability Estimates. With centered small ball probability calculation, bounds for shifted small ball probability are readily available if the bounds of the decentering part are obtained. Lemma 5.3 and Lemma 5.4 give upper and lower bounds of the decentering part as a function of rescaling level and model index parameter. Lemma 5.3 is used to lower bound the prior mass on $\left\{\left\|W^{a, \gamma_{n}^{*}}-f_{n}^{*}\right\|_{L_{2}\left(Q_{n}\right)}<\varepsilon_{n}\right\}$; Lemma 5.4 is used to upper bound the prior mass on $\left\{\Gamma \in \operatorname{FP}\left(\gamma_{n}^{*}\right)\right\} \cap\left\{\left\|W^{a, \Gamma}-f_{n}^{*}\right\|_{L_{2}\left(Q_{n}\right)}<\varepsilon_{n}\right\}$.

LEMmA 5.3. Suppose $\mathcal{H}^{a, \gamma_{0}}$ is the RKHS of the GP SEGP $\left(\mathbb{R}^{d_{n}} ; \gamma_{n}^{*}, \delta_{a}\right)$ with the design measure $Q_{n} \equiv N\left(0, \xi^{2} I_{d_{n}}\right), \gamma_{n}^{*} \in\{0,1\}^{d_{n}}$ encodes the indices of true variables, $d_{0} \equiv\left|\gamma_{n}^{*}\right|$, $f_{0} \in H^{\beta}\left(\mathbb{R}^{d_{0}}\right) \cap L_{2}\left(Q_{0}\right), f_{n}^{*}=T_{\gamma_{n}^{*}} f_{0}$, then for every $a>0$, there exists a constant $C$, $\varepsilon_{0}$ such that for all $\varepsilon<\varepsilon_{0}$

$$
\inf _{h \in \mathcal{H}^{a, \gamma_{0}}:\left\|h-f_{n}^{*}\right\|_{L_{2}\left(Q_{n}\right)}<\varepsilon}\|h\|_{\mathcal{H}^{a, \gamma_{0}}}^{2} \leq C(2 \sqrt{\pi})^{d_{0}} a^{d_{0}} e^{C \varepsilon^{-2 / \beta} / a^{2}} .
$$


The proof of Lemma 5.3 leverages the representation theorem of the RKHS and uses the squared RKHS norm of a special element in the true model neighborhood as an upper bound. In light of Lemma 4.1 of [29] and Lemma 7.1 of [28], after embedded into $L_{2}\left(Q_{n}\right)$, elements in $\mathcal{H}^{a, \gamma}$ admit the representation: for $t \in \mathbb{R}^{d_{n}}, h_{\psi}(t)=\int_{\mathbb{R}^{|\gamma|}} e^{i\left(\lambda, t_{0}\right)} \psi(\lambda) m_{a, \gamma}(\lambda) d \lambda$, where $t \equiv\left(t_{0}, t_{1}\right)$ with $t_{0} \in \mathbb{R}^{|\gamma|}$ and $t_{1} \in \mathbb{R}^{d_{n}} \backslash \mathbb{R}^{|\gamma|}, m_{a, \gamma}(\cdot)$ is the spectral density of the $\gamma$-dimensional Gaussian process with rescaling level $a$, and $\psi$ is in the complex Hilbert space $L_{2}\left(m_{a, \gamma}\right)$; its RKHS norm is defined as $\left\|h_{\psi}\right\|_{\mathcal{H}^{a, \gamma}}^{2}=\int_{\mathbb{R}^{|\gamma|} \mid}|\psi(\lambda)|^{2} m_{a, \gamma}(\lambda) d \lambda$. The spectral density $m_{a, \gamma}(\lambda)=a^{-|\gamma|} m_{\gamma}(\lambda / a)$, where $m_{\gamma}(\lambda)=e^{-\|\lambda\|_{2}^{2} / 4} /\left(2^{|\gamma|} \pi^{|\gamma| / 2}\right)$ and $\lambda \in$ $\mathbb{R}^{|\gamma|}$.

LEMMA 5.4. Suppose $\mathcal{H}^{a, \gamma}$ is the RKHS of the GP SEGP $\left(\mathbb{R}^{d_{n}} ; \gamma, \delta_{a}\right)$ with the design measure $Q_{n} \equiv N\left(0, \xi^{2} I_{d_{n}}\right), \gamma_{n}^{*} \in\{0,1\}^{d_{n}}$ encodes the indices of true variables, $d_{0} \equiv\left|\gamma_{n}^{*}\right|$, $f_{0} \in H^{\beta}\left(\mathbb{R}^{d_{0}}\right) \cap L_{2}\left(Q_{0}\right)$ with Fourier transform satisfying $\left|\widehat{f_{0} \sqrt{g_{d_{0}}}}(\lambda)\right| \succsim\|\lambda\|_{2}^{-\left(\alpha+d_{0} / 2\right)}$ for all $\|\lambda\|_{2} \geq 1$ and some constant $\alpha>0, f_{n}^{*}=T_{\gamma_{n}^{*}} f_{0}$, and $c_{\xi} \equiv \xi / \sqrt{2}$. Pick a $\gamma \in \operatorname{FP}\left(\gamma_{n}^{*}\right)$, then for all $a>0$, there exist constants $C, C^{\prime}$ and $\varepsilon_{0}$ only dependent of $f_{0}$, such that for all $\varepsilon<\varepsilon_{0}$,

$$
\inf _{h \in \mathcal{H}^{a, \gamma}:\left\|h-f_{n}^{*}\right\|_{L_{2}\left(Q_{n}\right)}<\varepsilon}\|h\|_{\mathcal{H}^{a, \gamma}}^{2} \geq C \varepsilon^{2}\left(c_{\xi} a\right)^{|\gamma|} e^{C^{\prime} \varepsilon^{-2 / \alpha}\left(\xi^{2} \wedge a^{-2}\right)} .
$$

The strategy of the proof follows the proof of Theorem 8 in [30]. In Lemma 5.4, the RKHS norm is expected to explode as the neighborhood in the false positive model space shrinks. Also note the effect of rescaling level $a$ : larger $a$ means better approximation and hence larger RKHS norm; small $a$ means flatness in the prior sample path and hence smaller RKHS norm.

6. Discussion. We have shown here that a GP regression model equipped with stochastic variable selection can simultaneously offer adaptive regression function estimation and consistent recovery of its sparsity pattern. This result is derived under several assumptions, some of which are mathematical formalizations of reasonable statistical considerations while others are needed more for technical reasons than statistical ones. Below we offer a detailed discussion of the reasonability and limitations of the formal assumptions and explore possible relaxations.

6.1. Gaussian design assumption. Assumption 1 requires a Gaussian random design and is quite restrictive, but is needed for a specific technical reason: it leads to a Karhunen-Loève eigen expansion of the squared exponential kernel in closed form. With the Karhunen-Loève expansion, the RKHS unit ball is isometric to an $\ell_{2}$ sequence space whose metric entropy can be accurately bounded. Further, small ball probability estimates are available via the metric entropy method $[18,20,28]$. In particular, it permits an upper bound of the prior mass condition that is crucial for Proposition 1. Also, the assumption $\xi^{2}>2 / e$ is made to simplify the calculations for Proposition 1. It holds in most applications as the design matrix can be standardized.

Without the Gaussian assumption, it is not tractable to work out sharp upper bounds of the prior mass condition needed for Proposition 1. An alternative approach to metric entropy calculation of the RKHS unit ball without assuming Gaussian random design is to extend [1] where the RKHS unit ball is identified as a set of "well-behaved" entire functions whose metric entropy can be accurately bounded. However, direct extension carrying over the rescaling parameter gives sub-optimal lower bounds of the metric entropy, and the resulting upper bounds of the prior mass condition become meaningless.

A natural relaxation is to assume the Radon-Nikodym derivative of $Q_{n}$ with respect to $G_{d_{n}}$ is bounded away from 0 and $\infty$, uniformly across all $n \geq 1$. This uniform absolute continuity 
implies that convergence in $\|\cdot\|_{L_{2}\left(Q_{n}\right)}$ is equivalent to convergence in $\|\cdot\|_{L_{2}\left(G_{d_{n}}\right)}$. Clearly, the uniform boundedness assumption renders the relaxation quite limited.

6.2. Fixed sparsity assumption. Assumption 2 makes it clear that for all large $n$, the true function is sparse and the support size $d_{0}$ does not grow with sample size. While the latter condition may appear too restrictive, it is shown in [34] that in the case $d_{n}$ grows nearly exponentially in $n$, one cannot hope to consistently estimate a sparse, smooth regression function nonparametrically unless the true support size remains essentially constant.

Assumption 3 identifies the true sparsity level $d_{0}$ as the minimal support size for the sequence $\left(f_{n}^{*}: n \geq 1\right)$. Under this assumption, each of the $d_{0}$ inputs to $f_{0}$ results in a variability that is detectable in the $L_{2}$ topology. This is essentially a nonparametric version of the $\beta$-min assumption in sparse linear regression literature.

6.3. Limited smoothness assumption. Assumption 4 imparts only a limited amount of smoothness on $f_{0}$. The first part of the assumption requires true $f_{0}$ to be in the Sobolev space $H^{\beta}\left(\mathbb{R}^{d_{0}}\right)$ in which functions satisfy (4). Analogous to Hölder smoothness, functions in $H^{\beta}\left(\mathbb{R}^{d_{0}}\right)$ have square-integrable (weak) partial derivatives up to order $\beta$. The second part of Assumption 4 is adapted from [30] and combines the probability density of the random design. It encodes a lower bound for the regularity of $f_{0}$ in the spirit of the self-similarity assumption in [16, 7, 26, 24]. A direct consequence of the assumption is lower bounds for the RKHS norm of the functions in a $L_{2}$ neighborhood of the true function (Lemma 5.4). Further, the resulting lower bounds are necessary for upper bounding the prior mass condition in Proposition 1. The self-similarity assumption in the Gaussian sequence model can be written as the convolution of $\hat{f}_{0}$ and a sum of delta functions evaluated at some frequencies. This convolution reflects the fixed design nature of Gaussian sequence model. In contrast, with random design, it is reasonable to weigh "signals" at different frequencies differently.

Note that the decay rate of a convolution is bounded above by the sum of the decay rates of the functions convolved when they are in both $L_{1}$ and $L_{\infty}$. As $\widehat{\sqrt{g_{d_{0}}}}$ is a Gaussian function decaying exponentially, $\widehat{\sqrt{g_{d_{0}}}} \in L_{1} \cap L_{\infty}$. Part 1 implies $\hat{f}_{0} \in L_{1} \cap L_{\infty}$. Therefore, part 2, coupled with part 1 , requires $\left|\widehat{f_{0} \sqrt{g_{d_{0}}}}(\lambda)\right|$ to decay approximately at the same rate as $\left|\hat{f}_{0}(\lambda)\right|$ as $\|\lambda\| \rightarrow \infty$, that is, $\left|\hat{f}_{0}(\lambda)\right| \succsim\|\lambda\|_{2}^{-\left(\alpha+d_{0} / 2\right)}$ as $\|\lambda\| \rightarrow \infty$.

One concern is whether there exists an $f_{0}$ satisfying Assumption 4 . The answer is yes. As an example, let $\hat{f}_{0}(\lambda)=(1+\|\lambda\|)^{-r}$ with $r=\alpha+d_{0} / 2>\beta+d_{0} / 2$. Then $f_{0} \in H^{\beta}\left(\mathbb{R}^{d_{0}}\right)$ and $\widehat{f_{0 \sqrt{g_{d_{0}}}}}(\lambda)=\hat{f}_{0} * \widehat{\sqrt{g_{d_{0}}}}(\lambda) \geq \int_{\|t\| \leq 1} \hat{f}_{0}(\lambda-t) \widehat{\sqrt{g_{d_{0}}}}(t) d t \succsim(2+\|\lambda\|)^{-r} \asymp\|\lambda\|_{2}^{-\left(\alpha+d_{0} / 2\right)}$. More such functions could be constructed as long as the tail decay rate of $\hat{f}_{0}$ is maintained.

The limited smoothness assumption rules out true functions that are infinitely differentiable. This assumption is key to our proof strategy which exploits a polynomial slowdown in posterior contraction rate when spurious predictors are included in the model. Similar rate slowdown also manifests for infinitely smooth true functions, but the depreciation is only logarithmic [29]. While our proof strategy fails to exploit such subtler rate drops, variable selection consistency may still hold in these cases. Indeed, for Bayesian linear regression with variable selection, posterior contraction rates slow down only by a multiplicative constant when spurious variables are included, and yet variable selection consistency holds in such cases.

Curiously, the results presented in this paper could be applied to construct a modified nonparametric regression model with guaranteed variable selection consistency, no matter the level of smoothness of the true function. One can augment the design matrix with an additional, independent Gaussian "covariate" $Z$, and create a new response $Y_{i}^{\prime}=Y_{i}+g_{0}\left(Z_{i}\right)$ where $g_{0}$ is a known function of limited smoothness. Now, for the new design $(X, Z)$ and the 
modified response data $Y^{\prime}$, the underlying true function has limited smoothness, and hence our results guarantee asymptotically accurate recovery of the important coordinate variables of $X$ in addition to the the synthetic variable $Z$. However, the resulting posterior convergence rate may be slower than optimal if $f_{0}$ were smoother than $g_{0}$.

6.4. Restricted design dimension growth assumption. Assumption 6 restricts the applicability of our result only up to a design size $\log \left(d_{n}\right) \precsim n^{d_{0} /\left(2 \beta+d_{0}\right)}$. Consequently, we are restricted to $\log \left(d_{n}\right) \ll n$ when $\beta$ is large. This is a serious limitation because as shown in [11], with true sparsity fixed at $d_{0}$, variable selection consistency should hold for larger design sizes, up to the limit $\log \left(d_{n}\right)=O(n)$. Notice however that we prove results for a Bayesian estimation method that simultaneously infers the sparsity patter $\Gamma$ and the regression function $f$, and offers a near minimax optimal estimation of the latter by adapting to the true smoothness level. It is this adaptation that imposes the stricter bound on $d_{n}$, beyond which the estimation error rate is dominated by the variable selection penalty which does not worsen polynomially between correct selection and false positive selections.

It is unclear at the moment whether the Bayesian estimation model studied here, or any other model which offers smoothness adaptive function estimation, could actually achieve variable selection consistency with $d_{n}$ growing faster than our bound. However, if one were to sacrifice on adaptive function estimation, variable selection consistency may be achieved even when $n^{d_{0} /\left(2 \beta+d_{0}\right)} \ll \log \left(d_{n}\right) \precsim n$ under a variation of our GP regression model where the random rescaling component (Assumption 5) is replaced with a dimension-specific deterministic rescaling

$$
\pi_{n, d}(a)=\mathbb{1}\left(a=n^{1 /(2 \underline{\beta}+d)}\right),
$$

for every $n \in \mathbb{N}, d \in\left\{1, \ldots, d_{n}\right\}$, where $\underline{\beta}$ is a fixed, small positive scalar. This modification grants variable selection consistency up to design dimensions $d_{n}$ with $\log \left(d_{n}\right) \precsim n^{d_{0} /\left(2 \underline{\beta}+d_{0}\right)}$ as long as the true smoothness $\beta>\underline{\beta}$. However, the posterior contraction rate under the deterministically rescaled GP prior is $O\left(n^{-\underline{\beta} /\left(2 \underline{\beta}+d_{0}\right)}\right)$ (up to a logarithmic factor of $n$ ), which could be much slower than the optimal rate $n^{-\beta /\left(2 \beta+d_{0}\right)}$. That is, in the extreme case $\log \left(d_{n}\right) \precsim n$, we can pick a small positive $\beta$ for the deterministic rescaling to guarantee variable selection consistency at the cost of estimation accuracy.

6.5. Separating variable selection from estimation. The discussion in the above two subsections indicates that one could gain on variable selection consistency by sacrificing on optimal function estimation. This leads to a much broader question. Should the two inference goals, optimal function estimation and consistent variable selection, be separated, and perhaps approached in a two-stage manner? We believe the answer is yes. A two-stage approach may sound contradictory to our adopted position of Bayesian modeling and inference. But in reality, the Bayesian paradigm, when augmented with decision theoretic considerations, may offer an incredibly fertile ground for exploring a rigorous two-stage approach. For example, one may adapt [17] and estimate $\gamma$ by the formal Bayes estimate $\hat{\gamma}_{B}$ under the loss function $L((f, \gamma),(\hat{f}, \hat{\gamma}))=\|f-\hat{f}\|_{L_{2}\left(Q_{n}\right)}^{2}+\lambda J(\hat{\gamma})$, for some model complexity penalty function $J(\gamma)$ and penalty tuning parameter $\lambda>0$. The Bayes estimate may be computed as $\hat{\gamma}_{B}=\arg \min _{\gamma}\left\{\left\|\bar{f}-\bar{f}_{\gamma}\right\|_{L_{2}\left(Q_{n}\right)}^{2}+\lambda J(|\gamma|)\right\}$ where $\bar{f}$ is the posterior mean of $f$ and $\bar{f}_{\gamma}=\int \bar{f}(x) \prod_{j: \gamma_{j}=0} G_{1}\left(d x_{j}\right)$ is the projection of $\bar{f}$ along $\gamma$ under $L_{2}\left(Q_{n}\right)$. Whether such computations are feasible and result in consistent estimation of $\gamma$ remain to be seen. It seems likely that the additional penalty component may help resolve the true model from false positive models, even if the posterior weights assigned to the latter were not polynomially smaller. 
Besides potential theoretical gains, such a Bayesian decision theoretic approach is appealing on philosophical grounds alone. Any statistical analysis may have any number of inference goals and no single estimation method may be universally optimal. For example, cross-validation based LASSO is known to offer great prediction accuracy while suffering from enhanced false detection [5]. A Bayesian decision theoretic approach may help unify such disparate goals where the analyst fits a single model to the data, but produces different estimates for different quantities by utilizing appropriately chosen loss function for each task. For example, in the adaptation described above, one may still report the posterior mean $\bar{f}$ as the Bayes estimate of $f$ (under the integrated square loss) while producing a sparse estimate $\hat{\gamma}_{B}$ for variable selection according the loss function discussed above. It will be exciting to carry out rigorous posterior asymptotic behavior of such formal Bayes estimates.

\section{REFERENCES}

[1] Aurzada, F., Ibragimov, I. A., Lifshits, M. and van Zanten, J. (2009). Small deviations of smooth stationary Gaussian processes. Theory of Probability \& Its Applications 53 697-707.

[2] Barron, A., Schervish, M. J. and Wasserman, L. (1999). The consistency of posterior distributions in nonparametric problems. The Annals of Statistics 27 536-561.

[3] Bertin, K. and Lecué, G. (2008). Selection of variables and dimension reduction in high-dimensional non-parametric regression. Electronic Journal of Statistics 2 1224-1241.

[4] Bhattacharya, A., Pati, D. and Dunson, D. (2014). Anisotropic function estimation using multibandwidth Gaussian processes. Annals of statistics $\mathbf{4 2} 352$.

[5] Bogdan, M., Van Den Berg, E., Sabatti, C., Su, W. and Candès, E. J. (2015). SlOPE-adaptive variable selection via convex optimization. The annals of applied statistics 91103.

[6] BÜHLmann, P. and VAn DE GeER, S. (2011). Statistics for high-dimensional data: methods, theory and applications. Springer Science \& Business Media.

[7] Bull, A. D. et al. (2012). Honest adaptive confidence bands and self-similar functions. Electronic Journal of Statistics 6 1490-1516.

[8] Butucea, C. and Stepanova, N. (2017). Adaptive variable selection in nonparametric sparse additive models. Electronic Journal of Statistics 11 2321-2357.

[9] CAstillo, I. (2008). Lower bounds for posterior rates with Gaussian process priors. Electronic Journal of Statistics 2 1281-1299.

[10] Castillo, I., Schmidt-Hieber, J. and VAn der VAart, A. (2015). Bayesian linear regression with sparse priors. The Annals of Statistics 43 1986-2018.

[11] Comminges, L. and Dalalyan, A. S. (2012). Tight conditions for consistency of variable selection in the context of high dimensionality. The Annals of Statistics 40 2667-2696.

[12] Ghosal, S., Ghosh, J. K. and VAn Der VAART, A. W. (2000). Convergence rates of posterior distributions. Annals of Statistics 28 500-531.

[13] Ghosal, S., Lember, J. and VAn Der VAart, A. (2008). Nonparametric Bayesian model selection and averaging. Electronic Journal of Statistics 2 63-89.

[14] GHosal, S. and VAN DER VAART, A. (2007). Convergence rates of posterior distributions for noniid observations. The Annals of Statistics 35 192-223.

[15] Ghosal, S. and VAn DER VAART, A. (2017). Fundamentals of nonparametric Bayesian inference 44. Cambridge University Press.

[16] Giné, E. and NickL, R. (2010). Confidence bands in density estimation. The Annals of Statistics 381122 1170.

[17] Hahn, P. R. and CARvalho, C. M. (2015). Decoupling shrinkage and selection in Bayesian linear models: a posterior summary perspective. Journal of the American Statistical Association 110 435-448.

[18] Kuelbs, J. and Li, W. V. (1993). Metric entropy and the small ball problem for Gaussian measures. Journal of Functional Analysis 116 133-157.

[19] LAFFERTY, J. and WASSERMAN, L. (2008). Rodeo: sparse, greedy nonparametric regression. The Annals of Statistics 36 28-63.

[20] LI, W. V. and LindE, W. (1999). Approximation, metric entropy and small ball estimates for Gaussian measures. The Annals of Probability 27 1556-1578.

[21] NARISETty, N. N. and HE, X. (2014). Bayesian variable selection with shrinking and diffusing priors. The Annals of Statistics 42 789-817.

[22] Raskutti, G., Wainwright, M. J. and YU, B. (2012). Minimax-optimal rates for sparse additive models over kernel classes via convex programming. Journal of Machine Learning Research 13 389-427. 
[23] Rasmussen, C. E. and Williams, C. K. (2006). Gaussian processes for machine learning 1. MIT press Cambridge.

[24] RAY, K. (2017). Adaptive Bernstein-von Mises theorems in Gaussian white noise. The Annals of Statistics 45 2511-2536.

[25] Stone, C. J. (1982). Optimal global rates of convergence for nonparametric regression. The annals of statistics $1040-1053$.

[26] Szabó, B., VAn Der VAART, A. W. and VAN ZANTEN, J. (2015). Frequentist coverage of adaptive nonparametric Bayesian credible sets. The Annals of Statistics 43 1391-1428.

[27] TOKDAR, S. T. (2011). Dimension adaptability of Gaussian process models with variable selection and projection. arXiv preprint arXiv:1112.0716.

[28] VAN DER VAART, A. W. and VAn ZANTEN, J. H. (2008). Reproducing kernel Hilbert spaces of Gaussian priors. In Pushing the limits of contemporary statistics: contributions in honor of Jayanta K. Ghosh 200-222. Institute of Mathematical Statistics.

[29] VAn Der VAart, A. W. and VAn Zanten, J. H. (2009). Adaptive Bayesian estimation using a Gaussian random field with inverse Gamma bandwidth. The Annals of Statistics 2655-2675.

[30] VAN DER VAART, A. and VAN ZANTEN, H. (2011). Information rates of nonparametric Gaussian process methods. Journal of Machine Learning Research 12 2095-2119.

[31] VerzelEn, N. (2012). Minimax risks for sparse regressions: Ultra-high dimensional phenomenons. Electronic Journal of Statistics 6 38-90.

[32] WAINWRIGHT, M. J. (2009). Information-theoretic limits on sparsity recovery in the high-dimensional and noisy setting. IEEE Transactions on Information Theory 55 5728-5741.

[33] Wainwright, M. J. (2019). High-dimensional statistics: A non-asymptotic viewpoint 48. Cambridge University Press.

[34] YAng, Y. and Tokdar, S. T. (2015). Minimax-optimal nonparametric regression in high dimensions. The Annals of Statistics $\mathbf{4 3}$ 652-674.

[35] Yang, Y., Wainwright, M. J. and Jordan, M. I. (2016). On the computational complexity of highdimensional Bayesian variable selection. The Annals of Statistics 44 2497-2532.

[36] ZhANG, P. (1991). Variable selection in nonparametric regression with continuous covariates. The Annals of Statistics 1869-1882.

\section{SUPPLEMENTARY MATERIAL}

Supplement to "Variable selection consistency of Gaussian process regression". This Supplement contains additional results and proofs in the text. 
7. Supplement to "Variable selection consistency of Gaussian process regression". The supplement file contains complete proofs for Theorem 4.1, Proposition 1, Lemma 5.1, Lemma 5.3, Lemma 5.4 in the paper "Variable selection consistency of Gaussian process regression".

\subsection{Proof of Theorem 4.1.}

ProOF. It suffices to show there exist sets (sieve) $\mathbb{B}_{n}$, such that the following three conditions hold for all sufficiently large $n$ :

$$
\begin{aligned}
& \Pi_{n}\left(\left\|W^{A, \Gamma}-f_{n}^{*}\right\|_{L_{2}\left(Q_{n}\right)} \leq \varepsilon_{n}\right) \geq e^{-n \varepsilon_{n}^{2}} \\
& \Pi_{n}\left(W^{A, \Gamma} \notin \mathbb{B}_{n}\right) \leq e^{-4 n \varepsilon_{n}^{2}} \\
& \log N\left(\varepsilon_{n}, \mathbb{B}_{n},\|\cdot\|_{L_{2}\left(Q_{n}\right)}\right) \leq n \varepsilon_{n}^{2} .
\end{aligned}
$$

\section{Prior mass condition}

Assumption $\beta>d_{0} / 2$ implies $1 / \varepsilon_{n} \geq C_{H}(a \xi)^{d_{0} / 2}$ and $a \xi \log \left(1 / \varepsilon_{n}\right)>d_{0}$ hold for every $a \in\left[C\left(1 / \varepsilon_{n}\right)^{1 / \beta}, 2 C\left(1 / \varepsilon_{n}\right)^{1 / \beta}\right]$ with some constant $C$. We can apply Lemma 5.2 and Lemma 5.3 to obtain the following lower bound

$$
\begin{aligned}
& \Pi_{n}\left(\left\|W^{A, \gamma_{n}^{*}}-f_{n}^{*}\right\|_{L_{2}\left(Q_{n}\right)} \leq 2 \varepsilon_{n}\right) \\
\geq & \int_{K_{n}}^{2 K_{n}} \Pi_{n}\left(\left\|W^{a, \gamma_{n}^{*}}-f_{n}^{*}\right\|_{L_{2}\left(Q_{n}\right)} \leq 2 \varepsilon_{n} \mid a\right) \Pi_{n}(d a) \\
\geq & \int_{K_{n}}^{2 K_{n}} e^{-\phi_{f_{n}}^{a, \gamma_{n}^{*}}\left(\varepsilon_{n}\right)} \pi_{n}(a) d a \\
\geq & \int_{K_{n}}^{2 K_{n}} e^{-\left(C a^{d_{0}}+C^{\prime} a^{d_{0}} \log \left(a / \varepsilon_{n}\right)^{d_{0}+1}\right.} \pi_{n}(a) d a \\
\geq & e^{-C \varepsilon^{-d_{0} / \beta} \log \left(1 / \varepsilon_{n}\right)^{d_{0}+1}}
\end{aligned}
$$

where $K_{n}=C\left(1 / \varepsilon_{n}\right)^{1 / \beta}, \varepsilon_{n} \asymp n^{-1 /\left(2+d_{0} / \beta\right)}(\log n)^{\kappa_{1}}$ and $\kappa_{1}=\frac{d_{0}+1}{2+d_{0} / \beta}$ such that quantity in the exponent satisfies $\varepsilon_{n}^{-d_{0} / \beta} \log \left(1 / \varepsilon_{n}\right)^{d_{0}+1} \precsim n \varepsilon_{n}^{2}$. We can achieve

$$
\Pi_{n}\left(\left\|W^{A, \gamma_{n}^{*}}-f_{n}^{*}\right\|_{L_{2}\left(Q_{n}\right)} \leq \varepsilon_{n}\right) \geq e^{-\frac{1}{2} n \varepsilon_{n}^{2}}
$$

by choosing $\varepsilon_{n}$ to be a large multiple of $n^{-1 /\left(2+d_{0} / \beta\right)}(\log n)^{\kappa_{1}}$.

Therefore, with prior mass of model $\gamma_{n}^{*}$ satisfying $\Pi_{n}\left(\Gamma=\gamma_{n}^{*}\right)=\Pi_{n}\left(|\Gamma|=d_{0}\right)\left(\begin{array}{c}d_{n} \\ d_{0}\end{array}\right)^{-1} \geq$ $e^{-\frac{1}{2} n \varepsilon_{n}^{2}}$

$$
\begin{aligned}
& \Pi_{n}\left(\left\|W^{A, \Gamma}-f_{n}^{*}\right\|_{L_{2}\left(Q_{n}\right)} \leq \varepsilon_{n}\right) \\
\geq & \Pi_{n}\left(\Gamma=\gamma_{n}^{*}\right) \Pi_{n}\left(\left\|W^{A, \gamma_{n}^{*}}-f_{n}^{*}\right\|_{L_{2}\left(Q_{n}\right)} \leq \varepsilon_{n}\right) \\
\geq & e^{-n \varepsilon_{n}^{2}} .
\end{aligned}
$$

\section{Sieve construction}

The sieve $\left\{\mathbb{B}_{n}\right\}$ is constructed as

$$
\mathbb{B}_{n}=\bigcup_{\gamma \in\{0,1\}^{d_{n}}:|\gamma| \leq \underline{d}_{n}} \mathbb{B}_{n, \gamma}
$$

where $\underline{d}_{n}=C\left(n \varepsilon_{n}^{2}\right)^{1 / \rho}$ for some constant $C$ and $\mathbb{B}_{n, 0^{d_{n}}}=\left[-M_{n}, M_{n}\right]$; for $\gamma \neq 0^{d_{n}}$,

$$
\mathbb{B}_{n, \gamma}=M_{n} \sqrt{r_{n}} \mathcal{H}_{1}^{r_{n}, \gamma}+\varepsilon_{n} \mathbb{B}_{1, \gamma},
$$

where $\mathbb{B}_{1, \gamma}$ is the unit ball in the Banach space $T_{\gamma}^{d_{n}} L_{2}\left(\mathbb{R}^{|\gamma|}\right)$ indexed by $\gamma . M_{n}$, and $r_{n}$ are specified such that $M_{n}^{2} \asymp n \varepsilon_{n}^{2}$, and $r_{n}^{|\gamma|} \log |\gamma|+1(n) \asymp n \varepsilon_{n}^{2}$. The choice of $r_{n}$ depends on $\gamma$ but for ease of notation $\gamma$ is dropped. To apply Lemma 5.1 and Lemma 5.2, it requires $r_{n}^{|\gamma|} \precsim \varepsilon_{n}^{-2}$. Clearly, the choice of $r_{n}$ satisfies this requirement. 
Verifying condition $\Pi_{n}\left(W^{A, \Gamma} \notin \mathbb{B}_{n}\right) \leq e^{-4 n \varepsilon_{n}^{2}}$

For $\gamma=0^{d_{n}}$, the prior on the regression function is $N(0,1)$,

$$
\begin{aligned}
\Pi_{n}\left(W^{0^{d_{n}}} \notin \mathbb{B}_{n}\right) & \leq \Pi_{n}\left(W^{0^{d_{n}}} \notin \mathbb{B}_{n, 0^{d}}\right) \\
& =2\left(1-\Phi\left(M_{n}\right)\right) \\
& \leq \frac{2}{\sqrt{2 \pi} M_{n}} e^{-M_{n}^{2} / 2}
\end{aligned}
$$

where $\Phi$ denotes standard Normal cdf. By choosing $M_{n}$ to be a large multiple of $n \varepsilon_{n}^{2}$, $\Pi_{n}\left(W^{0^{d_{n}}} \notin \mathbb{B}_{n}\right) \leq e^{-4 n \varepsilon_{n}^{2}}$ holds for all sufficiently large $n$.

In light of Lemma 4.7 of [29], the nesting property

$$
M_{n} \mathcal{H}_{1}^{a, \gamma}+\varepsilon_{n} \mathbb{B}_{1, \gamma} \subseteq B_{n, \gamma}
$$

holds for every $a \in\left[1 / \xi, r_{n}\right]$. By Borell's inequality, for every $a \in\left[1 / \xi, r_{n}\right]$ and $\gamma \neq 0^{d_{n}}$,

$$
\begin{aligned}
\Pi_{n}\left(W^{a, \gamma} \notin \mathbb{B}_{n}\right) & \leq \Pi_{n}\left(W^{a, \gamma} \notin \mathbb{B}_{n, \gamma}\right) \\
& \leq \Pi_{n}\left(W^{a, \gamma} \notin M_{n} \mathcal{H}^{a, \gamma}+\varepsilon_{n} \mathbb{B}_{1, \gamma}\right) \\
& \leq 1-\Phi\left(\Phi^{-1}\left(e^{-\phi_{0}^{a, \gamma}\left(\varepsilon_{n}\right)}\right)+M_{n}\right) \\
& \leq 1-\Phi\left(\Phi^{-1}\left(e^{-\phi_{0}^{r_{n}, \gamma}\left(\varepsilon_{n}\right)}\right)+M_{n}\right)
\end{aligned}
$$

where last inequality is because $e^{-\phi_{0}^{a, \gamma}\left(\varepsilon_{n}\right)}=\Pi_{n}\left(\left\|W^{a, \gamma}\right\|_{L_{2}\left(Q_{n}\right)} \leq \varepsilon_{n}\right)$ is decreasing in $a$.

In light of Lemma 5.2, $M_{n} \geq 4 \sqrt{\phi_{0}^{r_{n}, \gamma}\left(\varepsilon_{n}\right)}$ holds for all sufficiently large $n$. Since $e^{-\phi_{0}^{r_{n}, \gamma}\left(\varepsilon_{n}\right)}<1 / 4$ holds for all small enough $\varepsilon_{n}$, then it follows $M_{n} \geq-2 \Phi^{-1}\left(e^{-\phi_{0}^{r_{n}, \gamma}\left(\varepsilon_{n}\right)}\right)$ and the above inequality is further upper bounded by

$$
1-\Phi\left(M_{n} / 2\right) \leq e^{-M_{n}^{2} / 8} .
$$

So for every $\gamma \in\{0,1\}^{d_{n}} \backslash 0^{d_{n}}$, the following

$$
\begin{aligned}
& \Pi_{n}\left(W^{A, \gamma} \notin \mathbb{B}_{n}\right) \\
\leq & \Pi_{n}\left(W^{A, \gamma} \notin \mathbb{B}_{n, \gamma}\right) \\
\leq & \int_{1 / \xi}^{r_{n}} \Pi_{n}\left(W^{a, \gamma} \notin M_{n} \mathcal{H}_{1}^{a, \gamma}+\varepsilon_{n} \mathbb{B}_{1, \gamma}\right) \pi_{n}(a \mid \gamma) d a+\Pi_{n}\left(A>r_{n} \mid \gamma\right) \\
\leq & e^{-M_{n}^{2} / 8}+e^{-C_{2} r_{n}^{|\gamma|} \log |\gamma|+1}\left(r_{n}\right)+C_{3} \log (|\gamma|) \\
\leq & \frac{1}{2} e^{-4 n \varepsilon_{n}^{2}}
\end{aligned}
$$

holds for all sufficiently large $n$. Hence,

$$
\begin{aligned}
\Pi_{n}\left(W^{A, \Gamma} \notin \mathbb{B}_{n}\right) & =\sum_{\gamma \in\{0,1\}^{d}} \Pi_{n}\left(W^{A, \gamma} \notin \mathbb{B}_{n}\right) \Pi_{n}(\Gamma=\gamma) \\
& \leq \frac{1}{2} e^{-4 n \varepsilon_{n}^{2}}+\sum_{\gamma \in\{0,1\}^{d_{n}}:|\gamma|>\underline{d}_{n}} \Pi_{n}(\Gamma=\gamma) \\
& \leq e^{-4 n \varepsilon_{n}^{2}}
\end{aligned}
$$

Verifying condition $\log N\left(\varepsilon_{n}, \mathbb{B}_{n},\|\cdot\|_{L_{2}\left(Q_{n}\right)}\right) \leq n \varepsilon_{n}^{2}$

Clearly, $N\left(\mathbb{B}_{n, 0^{d_{n}}}, \varepsilon_{n},\|\cdot\|_{L_{2}\left(Q_{n}\right)}\right)=2 M_{n} / \varepsilon_{n}$.

In light of Lemma 5.1, for $M_{n} \sqrt{r_{n}}>2 \varepsilon_{n}$, the metric entropy of $M_{n} \sqrt{r_{n}} \mathcal{H}_{1}^{r_{n}, \gamma}+\varepsilon_{n} \mathbb{B}_{1, \gamma}$ is bounded above:

$$
\begin{aligned}
& \log N\left(2 \varepsilon_{n}, M_{n} \sqrt{r_{n}} \mathcal{H}_{1}^{r_{n}, \gamma}+\varepsilon_{n} \mathbb{B}_{1, \gamma},\|\cdot\|_{L_{2}\left(Q_{n}\right)}\right) \\
\leq & \log N\left(\varepsilon_{n}, M_{n} \sqrt{r_{n}} \mathcal{H}_{1}^{r_{n}, \gamma},\|\cdot\|_{L_{2}\left(Q_{n}\right)}\right) \\
\precsim & r_{n}^{|\gamma|} \log \left(M_{n} \sqrt{r_{n}} \varepsilon_{n}^{-1}\right)^{|\gamma|+1} /|\gamma| ! .
\end{aligned}
$$

Since $\log \left(M_{n} \sqrt{r_{n}} \varepsilon_{n}^{-1}\right) \asymp \log \left(1 / \varepsilon_{n}\right) \asymp \log (n)$, the above metric entropy is further bounded above by

$$
\log N\left(2 \varepsilon_{n}, M_{n} \sqrt{r_{n}} \mathcal{H}_{1}^{r_{n}, \gamma}+\varepsilon_{n} \mathbb{B}_{1, \gamma},\|\cdot\|_{L_{2}\left(Q_{n}\right)}\right) \precsim r_{n}^{|\gamma|} \log \left(1 / \varepsilon_{n}\right)^{|\gamma|+1} \asymp n \varepsilon_{n}^{2} .
$$


The bound holds for all $\gamma$ due to the choice of $r_{n}$.

Then note the bound $\log \left(\sum_{i=1}^{n} x_{i}\right) \leq \log n+\log \left(\max _{i} x_{i}\right)$, and the bound $\log (x \vee y) \leq$ $\log (x)+\log (y)$ for $x, y>1$, it follows,

$$
\log N\left(\varepsilon_{n}, \mathbb{B}_{n},\|\cdot\|_{2}\right) \precsim \log \left(2 \underline{d}_{n}-1\right)+\frac{1}{2} n \varepsilon_{n}^{2}+\log \left(\frac{2 M_{n}}{\varepsilon_{n}}\right)
$$

where $\underline{d}_{n} \prec n \varepsilon_{n}^{2}$ by construction and $\varepsilon_{n}$ is some multiple of $n^{-1 /\left(2+d_{0} / \beta\right)} \log ^{\kappa}(n)$.

7.2. Proof of Proposition 1. Before the proof of Proposition 1, first review some basic properties of Lambert $W$ function. Lambert $W$ function defines the product log function:

$$
x e^{x}=y \Leftrightarrow x=W(y) .
$$

In the proof of Proposition 1, we need to solve an equation like $\left(x-c_{1}\right) e^{x}=c_{2}$ for $x$. With Lambert $W$ function, the solution is in closed form: $x^{*}=W\left(c_{2} e^{-c_{1}}\right)+c_{1}$. The following lemma is useful for our calculations; a proof can be found in the end of the Supplement.

LEMMA 7.1. The Lambert $W(\cdot)$ function is strictly increasing. If $x=W(y)>1$, then $y>e^{W(y)}>y / \log (y)$. Furthermore, if $x=W(y) \in(0,1]$, then $y \geq W(y) \geq y / e$.

With the above preparation, here comes the proof of Proposition 1.

\subsubsection{Proof of Proposition 1.}

Proof. As in Lemma 1 of [9], Lemma 1 in [14], or Lemma 5 in [2], it suffices to show for some $\varepsilon_{n}$ with $\varepsilon_{n} \rightarrow 0$ and $n \varepsilon_{n}^{2} \rightarrow \infty$, the following holds

$$
\frac{\Pi_{n}\left(\Gamma \in \mathrm{FP}\left(\gamma_{n}^{*}\right), E_{n}\right)}{\Pi_{n}\left(B_{n}\left(f_{n}^{*}, \varepsilon_{n}\right)\right)}=o\left(e^{-4 n \varepsilon_{n}^{2}}\right)
$$

where $E_{n}=\left\{f:\left\|f-f_{n}^{*}\right\|_{L_{2}\left(Q_{n}\right)} \leq M \varepsilon_{n}\right\}$ as defined in the main text, and $B_{n}\left(f_{n}^{*}, \varepsilon_{n}\right)=$ $\left\{f: K L\left(P_{f_{n}^{*}}, P_{f}\right) \leq \varepsilon_{n}^{2}, V_{2,0}\left(P_{f_{n}^{*}}, P_{f}\right) \leq \varepsilon_{n}^{2}\right\}$ is the KL neighborhood of the truth. To prove (15), we show the following:

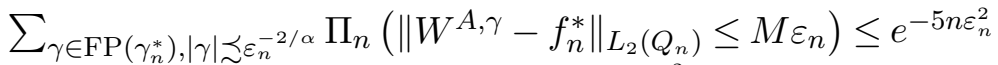

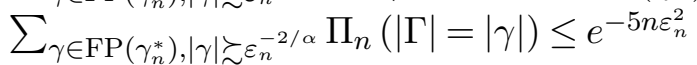

$$
\begin{aligned}
& \Pi_{n}\left(B_{n}\left(f_{n}^{*}, \varepsilon_{n}\right)\right) \geq \Pi_{n}\left(B_{n}\left(f_{n}^{*}, \varepsilon_{n}\right) \cap\left\{\Gamma=\gamma_{n}^{*}\right\}\right) \geq e^{-n \varepsilon_{n}^{2}}
\end{aligned}
$$

\section{Denominator}

In the nonparametric regression with Normal error, KL divergence is equivalent to $L_{2}\left(Q_{n}\right)$ norm: $K\left(P_{f_{n}^{*}}, P_{f}\right)=\frac{1}{2 \sigma^{2}}\left\|f_{n}^{*}-f\right\|_{L_{2}\left(Q_{n}\right)}^{2}$ and the KL variation $V_{2,0}\left(P_{f_{n}^{*}}, P_{f}\right)=\frac{1}{\sigma^{2}} \| f_{n}^{*}-$ $f \|_{L_{2}\left(Q_{n}\right)}^{2}$. Therefore, the denominator has the lower bound in $L_{2}\left(Q_{n}\right)$ norm:

$$
\Pi_{n}\left(B_{n}\left(f_{n}^{*}, \varepsilon_{n}\right)\right) \geq \Pi_{n}\left(\left\|W^{A, \gamma_{n}^{*}}-f_{n}^{*}\right\|_{L_{2}\left(Q_{n}\right)} \leq \sigma \varepsilon_{n}\right) \Pi_{n}\left(\Gamma=\gamma_{n}^{*}\right)
$$

where $\Pi_{n}\left(\Gamma=\gamma_{n}^{*}\right)$ is the prior probability of the true model. In light of the concentration inequality, Lemma 5.2 and Lemma 5.3,

$$
\begin{aligned}
& \Pi_{n}\left(\left\|W^{A, \gamma_{n}^{*}}-f_{n}^{*}\right\|_{L_{2}\left(Q_{n}\right)} \leq \sigma \varepsilon_{n}\right) \\
\geq & \int_{K_{n}}^{2 K_{n}} \Pi_{n}\left(\left\|W^{a, \gamma_{n}^{*}}-f_{n}^{*}\right\|_{L_{2}\left(Q_{n}\right)} \leq \sigma \varepsilon_{n} \mid a\right) \Pi_{n}(d a) \\
\geq & \int_{K_{n}}^{2 K_{n}} e^{-\phi_{f_{n}^{*}}^{a * \gamma_{n}^{*}}\left(\sigma \varepsilon_{n} / 2\right)} \Pi_{n}(d a) \\
\geq & \int_{K_{n}}^{2 K_{n}} e^{-\left(C a^{d_{0}}+C^{\prime} a^{d_{0}} \log \left(a / \varepsilon_{n}\right)^{d_{0}+1}\right)} \pi_{n}(a) d a \\
\geq & e^{-C \varepsilon_{n}^{-d_{0} / \beta} \log \left(1 / \varepsilon_{n}\right)^{d_{0}+1}}
\end{aligned}
$$


where $K_{n}=C\left(1 / \varepsilon_{n}\right)^{1 / \beta}, \varepsilon_{n} \asymp n^{-1 /\left(2+d_{0} / \beta\right)}(\log n)^{\kappa_{1}}$ and $\kappa_{1}=\frac{\left|\gamma_{n}^{*}\right|+1}{2+d_{0} / \beta}$. Note for $\gamma_{n}^{*}=0^{d_{n}}$, the prior on the mean is $W \sim N(0,1)$. Denote standard Normal density function as $\phi(\cdot)$, then

$$
\begin{aligned}
\Pi_{n}\left(\left\|W-f_{n}^{*}\right\|_{L_{2}\left(Q_{n}\right)} \leq \sigma \varepsilon_{n}\right) & =\Pi_{n}\left(f_{n}^{*}-\sigma \varepsilon_{n} \leq f \leq f_{n}^{*}+\sigma \varepsilon_{n}\right) \\
& \geq 2 \sigma \varepsilon_{n} \phi\left(\left(f_{n}^{*}+\sigma \varepsilon_{n}\right) \vee\left(f_{n}^{*}-\sigma \varepsilon_{n}\right)\right) \\
& \geq e^{-n \varepsilon_{n}^{2}}
\end{aligned}
$$

holds for every sufficiently large $n$.

Numerator

In light of the concentration inequality (13),

$$
\begin{aligned}
& \Pi_{n}\left(\left\|W^{A, \gamma}-f_{n}^{*}\right\|_{L_{2}\left(Q_{n}\right)} \leq M \varepsilon_{n}\right) \\
= & \int \Pi_{n}\left(\left\|W^{A, \gamma}-f_{n}^{*}\right\|_{L_{2}\left(Q_{n}\right)} \leq M \varepsilon_{n} \mid a\right) \Pi_{n}(d a \mid \gamma) \\
\leq & \int_{0}^{\infty} e^{-\phi_{f_{n}^{*}, \gamma}^{a, \gamma}\left(M \varepsilon_{n}\right)} \Pi_{n}(d a \mid \gamma),
\end{aligned}
$$

where $\Pi_{n}(\cdot \mid \gamma)$ denotes the prior on $A$ conditional on model index parameter $\gamma$. The sufficiently large constant $M$ can be absorbed into $\varepsilon_{n}$ by choosing it to be a large multiple of $n^{-1 /\left(2+d_{0} / \beta\right)}(\log n)^{\kappa_{1}}$. For the ease of notation, henceforth $M$ is absorbed into $\varepsilon_{n}$.

The integral (16) is further bounded by three parts:

$$
\int_{0}^{1 / \xi} e^{-\phi_{f_{n}^{*}}^{a, \gamma}\left(\varepsilon_{n}\right)} \Pi_{n}(d a \mid \gamma)+\int_{1 / \xi}^{\tau_{n}} e^{-\phi_{f_{n}^{*}}^{a, \gamma}\left(\varepsilon_{n}\right)} \Pi_{n}(d a \mid \gamma)+\Pi_{n}\left(A>\tau_{n} \mid \gamma\right)
$$

where $\tau_{n}=C \varepsilon_{n}{ }^{-1 / \beta}$ for some constant $C, \xi$ is a parameter of the dominating measure $Q_{n}$. The three quantities are bounded respectively as follows.

The first quantity of (17) is 0 due to the prior on $A$.

The third quantity of (17) has the desired upper bound $e^{-4 n \varepsilon_{n}^{2}}$, because $\tau_{n}^{|\gamma|} \succ n \varepsilon_{n}^{2}$ for every $\gamma \in F P\left(\gamma_{n}^{*}\right)$.

For the second quantity of (17), by Lemma 5.4 and Lemma 5.2, the integral is bounded above by

$$
D_{2} \tau_{n}^{|\gamma|} \sup _{a: 1 / \xi \leq a \leq \tau_{n}} \exp \left\{-C\left(c_{\xi} a\right)^{|\gamma|} \varepsilon_{n}^{2} e^{c \varepsilon_{n}^{-2 / \alpha} / a^{2}}-C_{2} a^{|\gamma|}+C_{3} \log (|\gamma|)\right\}
$$

where the prior density of $A \mid \Gamma=\gamma$ by assumption is upper bounded by $D_{2} \tau_{n}^{|\gamma|-1} e^{-C_{2} a^{|\gamma|}+C_{3} \log (|\gamma|)}$.

The solution to the supremum problem is $\tilde{a}=\sqrt{\frac{c_{n}}{W_{n}}}$ where $c_{n}=c \varepsilon_{n}^{-2 / \alpha}, C_{n}=$ $C_{2} C^{-1} c_{\xi}^{-|\gamma|} \varepsilon_{n}^{-2}, W_{n}=W\left(C_{n} e^{-|\gamma| / 2}|\gamma| / 2\right)+|\gamma| / 2$, and $W$ is Lambert $W$ function. This is solved by solving first order condition and verifying second order condition. Then the quantity (18) is proportional to

$$
\tau_{n}^{|\gamma|} \exp \left\{-C \varepsilon_{n}^{2}\left(c_{\xi} \tilde{a}\right)^{|\gamma|}\left(e^{W_{n}}+C_{n}\right)+C_{3} \log (|\gamma|)\right\} .
$$

In light of Lemma 7.1, there are two cases for $W(\cdot)$ and $W_{n}$. To apply Lemma 7.1, we need to solve $C_{n} e^{-|\gamma| / 2}|\gamma| / 2=e$ for $|\gamma|$. When $c_{\xi}^{2} e \leq 1, C_{n} e^{-|\gamma| / 2}|\gamma| / 2 \geq e$ holds for all $|\gamma|>d_{0}$. When $c_{\xi}^{2} e>1$, there exists only one solution larger than $d_{0}$ for all sufficiently large $n>$ $N\left(d_{0}, \xi\right)$. The solution is upper bounded by $\bar{\zeta}_{n} \equiv \frac{4}{1+2 \log c_{\xi}}(1+\Delta) \log \left(C_{2} C^{-1} \varepsilon_{n}^{-1}\right)$ for any constant $\Delta>0$, and is lower bounded by $\underline{\zeta}_{n} \equiv \frac{4}{1+2 \log c_{\xi}} \log \left(C_{2} C^{-1} \varepsilon_{n}^{-1}\right)$ for all sufficiently large $n>N(\Delta) \vee N\left(d_{0}, \xi\right)$. 
The followup analysis is divided into three regimes. Regime I deals with the fixed dimension and slowly growing dimension case. Regime II and regime III take care of growing dimension cases with different growth rates.

Regime I: $W\left(C_{n} e^{-|\gamma| / 2}|\gamma| / 2\right) \geq 1$.

In this regime, $C_{n} e^{-|\gamma| / 2}|\gamma| / 2 \geq e$ and $d_{0}<|\gamma|<\bar{\zeta}_{n} \asymp \log \left(\varepsilon_{n}^{-1}\right)$. By Lemma 7.1,

$$
\frac{1}{2}|\gamma| C_{n}>e^{W_{n}}>\frac{1}{2}|\gamma| C_{n} /\left(\log \left(|\gamma| C_{n} / 2\right)\right),
$$

and $\log \left(C_{n}\right)+\log (|\gamma|) \succsim W_{n} \succsim \log \left(C_{n}\right)+\log (|\gamma|)-\log \left(\log \left(C_{n}\right)+\log (|\gamma| / 2)\right)$. Then the supremum (19) is upper bounded by

$$
\exp \left\{-C_{2}\left(\frac{\varepsilon_{n}^{-2 / \alpha}}{\log C_{n}+\log (|\gamma|)}\right)^{|\gamma| / 2}+|\gamma| \log \left(\tau_{n}\right)+C_{3} \log (|\gamma|)\right\} .
$$

Note $\bar{\zeta}_{n}>|\gamma| \geq d_{0}+1$, the integral (18) is further bounded above by

$$
D_{2} \exp \left\{-C_{2}^{\prime}\left(\varepsilon_{n}^{-2 / \alpha} \log ^{-1}\left(\varepsilon_{n}^{-1}\right)\right)^{\left(d_{0}+1\right) / 2}+\bar{\zeta}_{n} \log \left(\tau_{n}\right)+C_{3} \log \left(\bar{\zeta}_{n}\right)\right\}
$$

for some constant $C_{2}^{\prime}$ and all sufficiently large $n$.

Since $\alpha<\beta\left(1+1 / d_{0}\right)$ and $\bar{\zeta}_{n} \asymp \log \tau_{n} \asymp \log \left(\varepsilon_{n}^{-1}\right)$, the following holds for all sufficiently large $n$

$$
\begin{aligned}
& \sum_{\gamma \in \mathrm{FP}\left(\gamma_{n}^{*}\right): W\left(C_{n} e^{-|\gamma| / 2}|\gamma| / 2\right) \geq 1} \Pi_{n}\left(\left\|W^{A, \gamma}-f_{n}^{*}\right\|_{L_{2}\left(Q_{n}\right)} \leq M \varepsilon_{n}\right) \\
\leq & 2^{\bar{\zeta}_{n}-d_{0}} D_{2} e^{-C_{2}^{\prime \prime}\left(\varepsilon_{n}^{-2 / \alpha} \log ^{-1}\left(\varepsilon_{n}^{-1}\right)\right)^{\left(d_{0}+1\right) / 2}} \\
\leq & \frac{1}{2} e^{-5 n \varepsilon_{n}^{2}} .
\end{aligned}
$$

Regime II: $W\left(C_{n} e^{-|\gamma| / 2}|\gamma| / 2\right)<1$ and $|\gamma| \precsim \varepsilon_{n}^{-2 / \alpha}$

In this regime, $C_{n}<e^{|\gamma| / 2+1}|\gamma| / 2,|\gamma|>\underline{\zeta}_{n}$, and $|\gamma| / 2 \leq W_{n} \leq|\gamma| / 2+1$. Then plugging in bounds on $W_{n}$, the supremum in (19) is upper bounded by $\exp \left\{-C_{2}\left(\frac{\varepsilon_{n}^{-2 / \alpha}}{|\gamma| / 2+1}\right)^{|\gamma| / 2}+|\gamma| \log \left(\tau_{n}\right)+C_{3} \log (|\gamma|)\right\}$. Note $\varepsilon_{n}^{-2 / \alpha} \succsim|\gamma|>\underline{\zeta}_{n}>d_{0}$, the integral (18) is further bounded above by

$$
\exp \left\{-C_{2}^{\prime}\left(\varepsilon_{n}^{-2 / \alpha} /\left(\underline{\zeta}_{n} / 2\right)\right)^{\underline{\zeta}_{n} / 2}+\varepsilon_{n}^{-2 / \alpha} \log \left(\tau_{n}\right)+C_{3}^{\prime} \log \left(\varepsilon_{n}^{-1}\right)\right\}
$$

for some constant $C_{2}^{\prime}$ and all sufficiently large $n$.

Since $\alpha<\beta\left(1+1 / d_{0}\right),|\gamma| \precsim \varepsilon_{n}^{-2 / \alpha} \prec n \varepsilon_{n}^{2}$, and $\underline{\zeta}_{n} \asymp \log \tau_{n} \asymp \log \left(\varepsilon_{n}^{-1}\right)$, the following holds for all sufficiently large $n$

$$
\begin{aligned}
& \sum_{\gamma \in \mathrm{FP}\left(\gamma_{n}^{*}\right): W\left(C_{n} e^{-|\gamma| / 2}|\gamma| / 2\right)<1} \Pi_{n}\left(\left\|W^{A, \gamma}-f_{n}^{*}\right\|_{L_{2}\left(Q_{n}\right)} \leq M \varepsilon_{n}\right) \\
\leq & 2^{C \varepsilon_{n}^{-2 / \alpha}}-\underline{\zeta}_{n} D_{2} e^{-C_{2}^{\prime}\left(\varepsilon_{n}^{-2 / \alpha} /\left(\underline{\zeta}_{n} / 2\right)\right)^{\underline{\zeta}_{n} / 2}} \\
\leq & \frac{1}{2} e^{-5 n \varepsilon_{n}^{2}} .
\end{aligned}
$$

Regime III: $W\left(C_{n} e^{-|\gamma| / 2}|\gamma| / 2\right)<1$ and $|\gamma| \succsim \varepsilon_{n}^{-2 / \alpha}$

In this regime, $\Pi_{n}(|\Gamma|=|\gamma|) \leq e^{-C|\gamma|^{\rho}}$ for some $\rho \geq\left(d_{0}+1\right) / 2$, the following holds for some constant $C^{\prime}$ and all sufficiently large $n$

$$
\Pi_{n}\left(\Gamma \in \mathrm{FP}\left(\gamma_{n}^{*}\right):|\Gamma| \geq \varepsilon_{n}^{-2 / \alpha}\right) \leq \Pi_{n}\left(|\Gamma| \geq \varepsilon_{n}^{-2 / \alpha}\right) \leq e^{-C^{\prime} \varepsilon_{n}^{-2 \rho / \alpha}} \leq e^{-5 n \varepsilon_{n}^{2}} .
$$

Therefore, combining the three regimes and the rest ingredients,

$$
\Pi_{n}\left(\Gamma \in \operatorname{FP}\left(\gamma_{n}^{*}\right),\left\{\left\|W^{A, \Gamma}-f_{n}^{*}\right\|_{L_{2}\left(Q_{n}\right)} \leq M \varepsilon_{n}\right\}\right) \leq 2 e^{-5 n \varepsilon_{n}^{2}}
$$

and the ratio (15) holds. 


\subsection{Proof of Lemma 5.1.}

PROOF. With some abuse of notation, denote (12) by $\mathcal{H}^{a, \gamma}$. By isometry, it suffices to compute the metric entropy of (12) with respect to the $\ell_{2}$ metric $\|\cdot\|_{2}$.

The strategy of the proof is to construct a $\tilde{\mathcal{H}} \subset \mathcal{H}^{a, \gamma}$ and use the metric entropy of $\tilde{\mathcal{H}}$ as bounds for the metric entropy of $\mathcal{H}^{a, \gamma}$. For the ease of notation, superscript " $(\gamma)$ " of the eigenvector and eigenvalue notation is dropped as the dependence on $\gamma$ is clear.

The eigenvalue $\mu_{j}$ takes the form $\left(2 v_{1} / V\right)^{|\gamma| / 2} B^{m}$ for some $m \in \mathbb{N}$. Solve

$$
\left(2 v_{1} / V\right)^{|\gamma| / 2} B^{m}=\varepsilon^{2}
$$

for $m \in \mathbb{R}$ and the solution denoted by $m^{*}$ is

$$
m^{*}=(\log (1 / B))^{-1}\left(2 \log (1 / \varepsilon)-\frac{|\gamma|}{2} \log \left(\frac{V}{2 v_{1}}\right)\right) .
$$

The assumption $1 / \varepsilon \geq C_{H}(\xi a)^{|\gamma| / 2}$ guarantees $m^{*} \asymp(\log (1 / B))^{-1} \log (1 / \varepsilon)$.

Define $\tau=\sum_{j=0}^{\left\lfloor m^{*}\right\rfloor}\left(\begin{array}{c}|\gamma|+j-1 \\ |\gamma|-1\end{array}\right)=\left(\begin{array}{c}\left\lfloor m^{*}\right\rfloor+|\gamma| \\ |\gamma|\end{array}\right)$ where $\left\lfloor m^{*}\right\rfloor$ is the greatest integer less than $m^{*}$. By construction, $\sqrt{\mu_{j}} \geq \varepsilon$ for all $j \leq \tau$ and $\sqrt{\mu_{j}} \leq \varepsilon$ for all $j>\tau$.

Define $\tilde{\mathcal{H}}_{\varepsilon}=\left\{\theta \in \mathcal{H}^{a, \gamma}: \theta_{j}=0, \forall j>\tau\right\}$. $\tilde{\mathcal{H}}_{\varepsilon}$ contains the elements in $\mathcal{H}^{a, \gamma}$ whose components after $\tau$ vanish to 0 . Any $\varepsilon$-cover of $\tilde{\mathcal{H}}_{\varepsilon}$ forms a $\sqrt{2} \varepsilon$-cover of $\mathcal{H}^{a, \gamma}$. To see this, suppose $\left\{\theta^{k}\right\}_{1}^{N}$ forms a $\varepsilon$-cover of $\tilde{\mathcal{H}}$, then for any $\theta \in \mathcal{H}^{a, \gamma}$,

$$
\begin{aligned}
\min _{k}\left\|\theta-\theta^{k}\right\|_{2}^{2} & =\min _{k} \sum_{j=1}^{\tau}\left(\theta_{j}-\theta_{j}^{k}\right)^{2}+\sum_{j=\tau+1}^{\infty} \theta_{j}^{2} \\
& \leq \varepsilon^{2}+\mu_{\tau} \sum_{j=\tau+1}^{\infty} \theta_{j}^{2} / \mu_{j} \\
& \leq 2 \varepsilon^{2} .
\end{aligned}
$$

As the $\sqrt{2}$ scaling does not matter in metric entropy calculation, it suffices to work with $\tilde{\mathcal{H}}$.

\section{Upper bound}

By construction, $\tilde{\mathcal{H}}_{\varepsilon} \supseteq B_{2}^{\tau+1}(\varepsilon)$ where $B_{q}^{k}(\varepsilon)$ denotes $k$-dimensional $\ell_{q}$-ball of radius $\varepsilon: B_{q}^{k}(\varepsilon)=\left\{x: \sum_{i=1}^{k} x_{i}^{q} \leq \varepsilon^{q} ; x_{j}=0, \forall j>k\right\} . \tau+1$ is due to eigenvalues start from $\mu_{0}$,

Note $\tilde{\mathcal{H}}_{\varepsilon}+B_{2}^{\tau+1}(\varepsilon) \subseteq 2 \tilde{\mathcal{H}}_{\varepsilon}$, standard volume argument yields

$$
N_{[]}\left(\varepsilon, \tilde{\mathcal{H}}_{\varepsilon},\|\cdot\|_{2}\right) \operatorname{vol}\left(B_{2}^{\tau+1}(\varepsilon / 2)\right) \leq \operatorname{vol}\left(2 \tilde{\mathcal{H}}_{\varepsilon}\right)
$$

where $\operatorname{vol}\left(2 \tilde{\mathcal{H}}_{\varepsilon}\right)=2^{\tau+1} \operatorname{vol}\left(\tilde{\mathcal{H}}_{\varepsilon}\right) \leq 2^{\tau+1} \prod_{i=0}^{\tau} \sqrt{\mu_{i}}$ and $\operatorname{vol}\left(B_{2}^{\tau+1}(\varepsilon / 2)\right)=(\varepsilon / 2)^{\tau+1} \operatorname{vol}\left(B_{2}^{\tau+1}(1)\right)$. Then, it follows

$$
\log N_{[]}\left(\varepsilon, \tilde{\mathcal{H}}_{\varepsilon},\|\cdot\|_{2}\right) \leq 2(\tau+1) \log 2+(\tau+1) \log (1 / \varepsilon)+\frac{1}{2} \sum_{i=0}^{\tau} \log \mu_{i}
$$

where $\log \left(\mu_{0}\right)=\frac{|\gamma|}{2} \log \left(2 v_{1} / V\right)$, and $\log \mu_{i}=\log \left(\mu_{0}\right)+h \log B$ for $h=1, \ldots,\left\lfloor m^{*}\right\rfloor$ and $i \in$ $\left[\left(\begin{array}{c}|\gamma|+h-1 \\ |\gamma|\end{array}\right),\left(\begin{array}{c}|\gamma|+h \\ |\gamma|\end{array}\right)\right)$. The exponent $h$ is the multiplicity of eigenvalues. lows

Note $B<1$ and total multiplicity is $\sum_{j=0}^{\left\lfloor m^{*}\right\rfloor} j\left(\begin{array}{c}|\gamma|-1+j \\ |\gamma|-1\end{array}\right) \geq \sum_{j=1}^{\left\lfloor m^{*}\right\rfloor}\left(\begin{array}{c}|\gamma|-1+j \\ |\gamma|-1\end{array}\right)=\tau-1$, it fol-

$$
\begin{aligned}
\sum_{i=0}^{\tau} \log \mu_{i} & =\frac{|\gamma|}{2} \log \left(2 v_{1} / V\right)(\tau+1)-\sum_{j=0}^{\left\lfloor m^{*}\right\rfloor} j\left(\begin{array}{c}
|\gamma|-1+j \\
|\gamma|-1
\end{array}\right) \log (1 / B) \\
& \leq \frac{|\gamma|}{2} \log \left(2 v_{1} / V\right)(\tau+1)-\log (1 / B)(\tau-1) .
\end{aligned}
$$


Note $a \xi \log (1 / \varepsilon)>|\gamma|$ implies $m^{*} \asymp m^{*}+|\gamma|$, the with the definition of $\tau$ and $1 / \varepsilon \geq$ $C_{H}(\xi a)^{|\gamma| / 2}$,

$$
\begin{aligned}
& \log N_{[]}\left(\varepsilon, \tilde{\mathcal{H}}_{\varepsilon},\|\cdot\|_{2}\right) \\
\precsim & (\tau+1)\left[\log (1 / \varepsilon)-\frac{|\gamma|}{4} \log \left(\frac{V}{2 v_{1}}\right)\right]-\frac{1}{2}(\tau-1) \log (1 / B) \\
\precsim & \left(m^{*}+|\gamma|\right)^{|\gamma|} \log (1 / \varepsilon) /|\gamma| ! \\
\precsim & (\log (1 / B))^{-|\gamma|} \log (1 / \varepsilon)^{|\gamma|+1} /|\gamma| ! .
\end{aligned}
$$

\section{Lower bound}

Since $\tilde{\mathcal{H}}_{\varepsilon} \subset \mathcal{H}^{a, \gamma}$, lower bound of $\tilde{\mathcal{H}}_{\varepsilon}$ 's metric entropy also lower bounds $\mathcal{H}^{a, \gamma}$ 's metric entropy. The fact $\tilde{\mathcal{H}}_{\varepsilon} \supseteq B_{2}^{\tau+1}(\varepsilon)$ implies

$$
\begin{aligned}
\log N\left(\tilde{\mathcal{H}}_{\varepsilon}, \varepsilon / 2,\|\cdot\|_{2}\right) & \geq \log N\left(B_{2}^{\tau+1}(\varepsilon), \varepsilon / 2,\|\cdot\|_{2}\right) \\
& \geq(\tau+1) \log 2 \\
& \asymp(\log (1 / B))^{-|\gamma|} \log (1 / \varepsilon)^{|\gamma|} /|\gamma|,
\end{aligned}
$$

and $\log N\left(\mathcal{H}^{a, \gamma}, \varepsilon,\|\cdot\|_{2}\right) \succsim(\log (1 / B))^{-|\gamma|} \log (1 / \varepsilon)^{|\gamma|} /|\gamma| !$.

Bounding $\log (1 / B)$

Note

$$
\begin{aligned}
\log (1 / B) & =\log \left(1+v_{1} / v_{2}+v_{3} / v_{2}\right) \\
& =\log \left(1+v_{1} / v_{2}+\sqrt{\left(v_{1} / v_{2}\right)^{2}+2 v_{1} / v_{2}}\right)
\end{aligned}
$$

where $v_{1} / v_{2}=1 /\left(4 a^{2} \xi^{2}\right)$.

When $a$ is close to $0, \log (1 / B) \asymp \log \left(v_{1} / v_{2}\right) \asymp \log (1 / a) \precsim 1 / a$.

When $a$ is sufficiently large, $v_{1} / v_{2}$ is close to 0 and $\sqrt{\left(v_{1} / v_{2}\right)^{2}+2 v_{1} / v_{2}} \succ v_{1} / v_{2}$. With the relation $\log (1+x) \approx x$ for $x \approx 0$, it follows $\log (1 / B)^{-1} \asymp a \xi$.

For more precise characterization, there exists constants $C_{1}, C_{2}$ and $C_{3}$ such that

$$
C_{1} a \leq \log (1 / B)^{-1} \leq C_{2}+C_{3} a
$$

holds for all $a>0$. The constants only depend on $\xi$.

\subsection{Proof of Lemma 5.3.}

PROOF. The proof extends Lemma 7 of [30] and allows for rescaling and different dimensions. Following the representation theorem, for $\lambda_{0} \in \mathbb{R}^{d_{0}}$, let

$$
\psi\left(\lambda_{0}\right)=\frac{\hat{f}_{0}\left(\lambda_{0}\right)}{m_{a, \gamma_{n}^{*}}\left(\lambda_{0}\right)} 1_{\left(\lambda_{0} \in \mathbb{R}^{d_{0}}:\left\|\lambda_{0}\right\|_{2}<K\right)},
$$

then for every $t \in \mathbb{R}^{d_{n}}, f_{n}^{*}(t)-h_{\psi}(t)=\int_{\lambda_{0} \in \mathbb{R}^{d_{0}}:\left\|\lambda_{0}\right\|_{2} \geq K} e^{i\left(\lambda_{0}, t_{0}\right)} \hat{f}_{0}\left(\lambda_{0}\right) d \lambda_{0}$. By Hölder 's inequality,

$$
\begin{aligned}
\left|h_{\psi}(t)-f_{n}^{*}(t)\right|^{2} & \leq \int_{\mathbb{R}^{d_{0}}}\left|\hat{f}_{0}\left(\lambda_{0}\right)\right|^{2} 1\left(\left\|\lambda_{0}\right\|_{2} \geq K\right) d \lambda_{0} \\
& \leq\left\|f_{0}\right\|_{H^{\beta}\left(\mathbb{R}^{d_{0}}\right)}^{2} \sup _{\lambda_{0} \in \mathbb{R}^{d_{0}}}\left(1+\left\|\lambda_{0}\right\|_{2}^{2}\right)^{-\beta} 1\left(\left\|\lambda_{0}\right\|_{2} \geq K\right) \\
& \precsim\left\|f_{0}\right\|_{H^{\beta}\left(\mathbb{R}^{d_{0}}\right)}^{2} K^{-2 \beta} .
\end{aligned}
$$


Therefore,

$$
\begin{aligned}
\left\|h_{\psi}-f_{n}^{*}\right\|_{L_{2}\left(Q_{n}\right)}^{2} & =\int_{\mathbb{R}^{d_{n}}}\left|h_{\psi}(t)-f_{n}^{*}(t)\right|^{2} g_{d_{n}}(t) d t \\
& \precsim\left\|f_{0}\right\|_{H^{\beta}\left(\mathbb{R}^{d_{0}}\right)}^{2} K^{-2 \beta} .
\end{aligned}
$$

Choose $K^{-\beta} \propto \varepsilon$ such that $\left\|h_{\psi}-f_{n}^{*}\right\|_{L_{2}\left(Q_{n}\right)} \leq \varepsilon$.

$$
\begin{aligned}
\left\|h_{\psi}\right\|_{\mathcal{H}^{a, \gamma_{n}^{*}}}^{2} & =\int_{\lambda_{0} \in \mathbb{R}^{d_{0}}:\left\|\lambda_{0}\right\|_{2}<K}\left|\hat{f}_{0}\left(\lambda_{0}\right)\right|^{2} m_{a, \gamma_{n}^{*}}\left(\lambda_{0}\right)^{-1} d \lambda_{0} \\
& =\int_{\lambda_{0} \in \mathbb{R}^{d_{0}}:\left\|\lambda_{0}\right\|_{2}<K}\left|\hat{f}_{0}\left(\lambda_{0}\right)\right|^{2}(2 \sqrt{\pi})^{d_{0}} a^{d_{0}} e^{\frac{1}{4 a^{2}}\left\|\lambda_{0}\right\|_{2}^{2}} d \lambda_{0} \\
& \leq\left\|f_{0}\right\|_{H^{\beta}\left(\mathbb{R}^{d_{0}}\right)}^{2}(2 \sqrt{\pi})^{d_{0}} a^{d_{0}} e^{\frac{1}{4 a^{2}} K^{2}} .
\end{aligned}
$$

Then it holds that

$$
\left\|h_{\psi}\right\|_{\mathcal{H}^{a, \gamma_{n}^{*}}}^{2} \precsim(2 \sqrt{\pi})^{d_{0}} a^{d_{0}} e^{C \varepsilon^{-2 / \beta} / a^{2}}
$$

\subsection{Proof of Lemma 5.4.}

PROOF. By Parseval's identity, for $h_{\psi} \in \mathcal{H}^{a, \gamma}$,

$$
\left\|h_{\psi}-f_{n}^{*}\right\|_{L_{2}\left(Q_{n}\right)}^{2}=\left\|\left(h_{\psi}-f_{n}^{*}\right) \sqrt{g_{d_{n}}}\right\|_{2}^{2}=\left\|\widehat{\mid h_{\psi \sqrt{g_{d_{n}}}}}-\widehat{f_{n}^{*} \sqrt{g_{d_{n}}}}\right\|_{2}^{2} .
$$

Therefore, $\left\|h_{\psi}-f_{n}^{*}\right\|_{L_{2}\left(Q_{n}\right)}<\varepsilon$ implies $\left\|\widehat{h_{\psi \sqrt{g_{d_{n}}}}} \chi_{K}-\widehat{f_{n}^{*} \sqrt{g_{d_{n}}}} \chi_{K}\right\|_{2}<\varepsilon$, where $\chi_{K}=$ $\left\{\lambda \in \mathbb{R}^{|\gamma|}:\|\lambda\|_{2}>K\right\}$. Triangle inequality implies

$$
\left\|\widehat{h_{\psi \sqrt{g_{d_{n}}}}} \chi_{K}\right\|_{2}>\left\|\widehat{f_{n}^{*} \sqrt{g_{d_{n}}}} \chi_{K}\right\|_{2}-\varepsilon
$$

For the right hand side of inequality (20), denote $\mathbb{R}^{|\gamma|} \backslash \mathbb{R}^{d_{0}}$ as $\mathbb{R}^{d_{1}}$, with the assumption on $f_{0}$,

$$
\begin{aligned}
& \|\left.\widehat{f_{n}^{*} \sqrt{g_{d_{n}}}} \chi_{K}\right|_{2} ^{2} \\
\propto & \iint_{\left(\lambda_{0}, \lambda_{1}\right) \in \mathbb{R}|\gamma|:\left.|| \lambda_{0}\left|\|_{2}^{2}+\right|\left|\lambda_{1}\right|\right|_{2} ^{2}>K^{2}}\left|\widehat{f_{0 \sqrt{g_{d_{0}}}}}\left(\lambda_{0}\right)\right|^{2}\left|\widehat{\sqrt{g_{d_{1}}}}\left(\lambda_{1}\right)\right|^{2} d \lambda_{0} d \lambda_{1} \\
\geq & \iint_{\left(\lambda_{0}, \lambda_{1}\right) \in \mathbb{R}^{|\gamma|}:\left\|\lambda_{0}\right\|_{2}>K}\left|\widehat{f_{0} \sqrt{g_{d_{0}}}}\left(\lambda_{0}\right)\right|^{2}\left|\widehat{\sqrt{g_{d_{1}}}}\left(\lambda_{1}\right)\right|^{2} d \lambda_{0} d \lambda_{1} \\
& \succsim \int_{\lambda \in \mathbb{R}_{0} d_{0}:\left\|\lambda_{0}\right\|_{2}>K}|| \lambda_{0} \|^{-\left(2 \alpha+d_{0}\right)} d \lambda_{0} \\
\succsim & (1 / K)^{2 \alpha}
\end{aligned}
$$

where $K \propto \varepsilon^{-1 / \alpha}$ is chosen such that $\left\|\widehat{f_{n}^{*} \sqrt{g_{d_{n}}}} \chi_{K}\right\|_{2}$ is lower bounded by $2 \varepsilon$.

By Lemma 16 of [30] and the representation of $h_{\psi}$,

$$
\begin{aligned}
& \left\|\widehat{h_{\psi \sqrt{g_{d_{n}}}}} \chi_{2 K}\right\|_{2} \\
= & \left\|\left(\left(\psi m_{a, \gamma}\right) * \widehat{\sqrt{g_{d_{n}}}}\right) \chi_{2 K}\right\|_{2} \\
\leq & \left\|\psi m_{a, \gamma} \chi_{K}\right\|_{2}\left\|\widehat{\sqrt{g_{d_{n}}}}\left(1-\chi_{K}\right)\right\|_{1}+\left\|\psi m_{a, \gamma}\right\|\left\|_{2}\right\| \widehat{\sqrt{g_{d_{n}}}} \chi_{K} \|_{1} .
\end{aligned}
$$

The terms on the above right hand side are bounded in the following way,

$$
\begin{aligned}
\left\|\psi m_{a, \gamma} \chi_{K}\right\|_{2}^{2} & =\int_{\lambda \in \mathbb{R}|\gamma|:\|\lambda\|_{2}>K}|\psi(\lambda)|^{2} m_{a, \gamma}(\lambda)^{2} d \lambda \\
\leq & m_{a, \gamma}(K) \int_{\lambda \in \mathbb{R}|\gamma|: \mid\|\lambda\|_{2}>K}|\psi(\lambda)|^{2} m_{a, \gamma}(\lambda) d \lambda \\
\leq & (2 \sqrt{\pi})^{-|\gamma|} a^{-|\gamma|} e^{-\frac{1}{4} K^{2} / a^{2}}\left\|h_{\psi}\right\|_{\mathcal{H}^{a, \gamma}}^{2} \\
\left\|\widehat{\sqrt{g_{d_{n}}}}\left(1-\chi_{K}\right)\right\|_{1} & =\int_{\lambda \in \mathbb{R}|\gamma|:|| \lambda \|_{2} \leq K}(2 \sqrt{2 \pi} \xi)^{|\gamma| / 2} e^{-\xi^{2}\|\lambda\|_{2}^{2}} d \lambda \\
& <\int_{\mathbb{R}|\gamma|}(2 \sqrt{2 \pi} \xi)^{|\gamma| / 2} e^{-\xi^{2}\|\lambda\|_{2}^{2}} d \lambda \\
& \precsim(2 \sqrt{2 \pi})^{|\gamma| / 2} \xi^{-|\gamma| / 2}<\infty
\end{aligned}
$$




$$
\begin{aligned}
&\left\|\psi m_{a, \gamma}\right\|_{2}^{2}=\int_{\mathbb{R}|\gamma|}|\psi(\lambda)|^{2} m_{a, \gamma}(\lambda)^{2} d \lambda \\
& \leq(2 \sqrt{\pi})^{-|\gamma|} a^{-|\gamma|} \int_{\mathbb{R}|\gamma|}|\psi(\lambda)|^{2} m_{a, \gamma}(\lambda) d \lambda \\
&=(2 \sqrt{\pi})^{-|\gamma|} a^{-|\gamma|}|| h_{\psi} \|_{\mathcal{H}^{a, \gamma}}^{2} \\
&\left\|\widehat{\sqrt{g_{d_{n}}}} \chi_{K}\right\|_{1}=\int_{\lambda \in \mathbb{R}|\gamma|: \mid\|\lambda\|_{2}>K}(2 \sqrt{2 \pi} \xi)^{|\gamma| / 2} e^{-\xi^{2}|| \lambda \|_{2}^{2}} d \lambda \\
& \leq e^{-\xi^{2} K^{2} / 8} \int_{\lambda \in \mathbb{R}|\gamma|:|| \lambda \|_{2}>K}(2 \sqrt{2 \pi} \xi)^{|\gamma| / 2} e^{-\frac{7}{8} \xi^{2}\|\lambda\|_{2}^{2}} d \lambda \\
& \leq e^{-\xi^{2} K^{2} / 8} \int(2 \sqrt{2 \pi} \xi)^{|\gamma| / 2} e^{-\frac{7}{8} \xi^{2}\|\lambda\|_{2}^{2}} d \lambda \\
& \precsim(2 \sqrt{2 \pi})^{|\gamma| / 2} \xi^{-|\gamma| / 2} e^{-\xi^{2} K^{2} / 8}
\end{aligned}
$$

Since a factor of 2 does not matter, combining bounds of RHS and LHS of inequality (20) yields the following inequality

$$
a^{-|\gamma| / 2}(\sqrt{2} / \xi)^{|\gamma| / 2}\left(e^{-\frac{1}{8} K^{2} / a^{2}}+e^{-\frac{1}{8} \xi^{2} K^{2}}\right)\left\|h_{\psi}\right\|_{\mathcal{H}^{a, \gamma}} \succsim\left\|\widehat{h_{\psi \sqrt{g_{d_{n}}}}} \chi_{2 K}\right\|_{2} \geq \varepsilon
$$

which is

$$
\left\|h_{\psi}\right\|_{\mathcal{H}^{a, \gamma}}^{2} \succsim \varepsilon^{2} a^{|\gamma|} c_{\xi}^{|\gamma|} e^{\frac{1}{4} K^{2}\left(a^{-2} \wedge \xi^{2}\right)}
$$

where $c_{\xi}=\xi / \sqrt{2}$.

\subsection{Proof of Lemma 7.1.}

Proof. Let $f(x)=x e^{x}$ and $f$ is a strictly increasing, smooth map: $[0, \infty) \rightarrow[0, \infty)$. So its inverse is also strictly increasing.

For the second claim, the first inequality follow because by the definition of $W(\cdot)$ and the assumption $x>1$,

$$
y=W(y) e^{W(y)}>e^{W(y)} .
$$

That is, $\log (y)>W(y)$. Then, $\log (y) e^{W(y)}>W(y) e^{W(y)}=y$, which is the second inequality.

Finally, as $W(y) \in(0,1]$, by monotonicity, $W(y) \leq W(y) e^{W(y)} \equiv y \leq W(y) e$, that is,

$$
y \geq W(y) \geq y / e .
$$

